\title{
Discrete two dimensional Fourier transform in polar coordinates Part II: numerical computation and approximation of the continuous transform
}

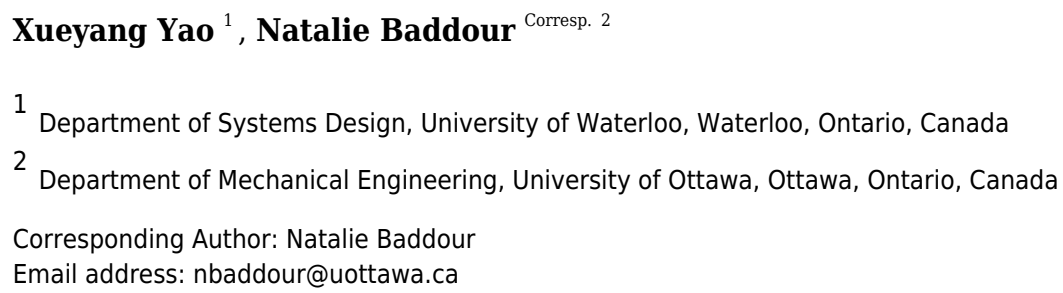

The theory of the continuous two-dimensional (2D) Fourier Transform in polar coordinates has been recently developed but no discrete counterpart exists to date. In the first part of this two-paper series, we proposed and evaluated the theory of the 2D discrete Fourier Transform (DFT) in polar coordinates. The theory of the actual manipulated quantities was shown, including the standard set of shift, modulation, multiplication, and convolution rules. In this second part of the series, we address the computational aspects of the 2D DFT in polar coordinates. Specifically, we demonstrate how the decomposition of the 2D DFT as a DFT, Discrete Hankel Transform (DHT) and inverse DFT sequence can be exploited for coding. We also demonstrate how the proposed 2D DFT can be used to approximate the continuous forward and inverse Fourier transform in polar coordinates in the same manner that the 1D DFT can be used to approximate its continuous counterpart. 
1 Discrete two dimensional Fourier transform in polar approximation of the continuous transform

Xueyang Yao $^{1}$, Natalie Baddour ${ }^{2}$

$5 \quad{ }^{1}$ Department of Systems Design, University of Waterloo, 200 University Avenue West, Waterloo,

6 Ontario, N2L 3G1, Canada

7 2Department of Mechanical Engineering, University of Ottawa, 161 Louis Pasteur, Ottawa,

8 Ontario, K1N 6N5, Canada

9 1xueyang.yao@uwaterloo.ca

10 2nbaddour@uottawa.ca (corresponding author)

\section{Abstract}

The theory of the continuous two-dimensional (2D) Fourier Transform in polar coordinates has been recently developed but no discrete counterpart exists to date. In the first part of this twopaper series, we proposed and evaluated the theory of the 2D discrete Fourier Transform (DFT) in polar coordinates. The theory of the actual manipulated quantities was shown, including the standard set of shift, modulation, multiplication, and convolution rules. In this second part of the series, we address the computational aspects of the 2D DFT in polar coordinates. Specifically, we demonstrate how the decomposition of the 2D DFT as a DFT, Discrete Hankel Transform (DHT) and inverse DFT sequence can be exploited for coding. We also demonstrate how the proposed 2D DFT can be used to approximate the continuous forward and inverse Fourier transform in polar coordinates in the same manner that the 1D DFT can be used to approximate its continuous counterpart.

\section{Introduction}

The Fourier Transform (FT) is a powerful analytical tool and has proved to be invaluable in many disciplines such as physics, mathematics and engineering. The development of the Fast Fourier Transform (FFT) algorithm [1], which computes the discrete Fourier transform with a fast algorithm, firmly established the Fourier transform as a practical tool in diverse areas, most notably signal and image processing.

In two dimensions, the FFT can still be used to compute the discrete Fourier transform in Cartesian coordinates. However, in many applications such as photoacoustics [2] and tomography [3]-[6], it is often necessary to compute the Fourier transform in polar coordinates. Moreover, for functions that are naturally described in polar coordinates, a discrete version of the 2D Fourier transform in polar coordinates is needed. There have been some attempts to calculate the Fourier transform in polar coordinates, most notably through the Hankel transform, since the zeroth order 
Hankel transform is known to be a 2D Fourier transform in polar coordinates for rotationally symmetric functions. However, prior work has focused on numerically approximating the continuous transform. This stands in contrast to the Fourier transform, where the Discrete Fourier Transform (DFT) can stand alone as an orthogonal transform, independent of the existence of its continuous counterpart.

The idea of a Polar Fourier transforms has been previously investigated, where the spatial function is in Cartesian coordinates but its FT is computed in polar coordinates [7]-[9]. Fourier transforms have been proposed for non-equispaced data, referred to as a Unequally Spaced FFT (USFFT) or Non-Uniform FFT (NUFFT) [10]-[14]. A recent book gives a unified treatment of these topics [15]. Previous work has also considered the implications of using a polar grid [16], [17]. Although the above references demonstrate that the computation of a discrete 2D FT on a polar grid has previously been considered in the literature, there is to date no discrete 2D Fourier transform in polar coordinates that exists as a transform in its own right, with its own set of rules of the actual manipulated quantities.

In part I of this two part series, we proposed an independent discrete 2D Fourier transform in polar coordinates, which has been defined to be discrete from first principles [18]. For a discrete transform, the values of the transform are only given as entries in a vector or matrix and the transform manipulates a set of discrete values. To quote Bracewell [19], "we often think of this as though an underlying function of a continuous variable really exists and we are approximating it. From an operational viewpoint, however, it is irrelevant to talk about the existence of values other than those given and those computed (the input and output). Therefore, it is desirable to have a mathematical theory of the actual quantities manipulated". Hence, in our previous paper [18], standard operational 'rules' of shift, modulation and convolution rules for this 2D DFT in polar coordinates were demonstrated. The operational rules were demonstrated via the key properties of the proposed discrete kernel of the transform. However, using the discrete kernel may not be the most effective way to compute the transform. Furthermore, while the 2D DFT in polar coordinates was demonstrated to have properties and rules as a standalone transform independent of its relationship to any continuous transform, an obvious application of the proposed discrete transform is to approximate its continuous counterpart.

Hence, the goal of this second part of this two-part paper series is to propose computational approaches to the computation of the previously proposed 2D DFT in polar coordinates and also to validate its effectiveness to approximate the continuous 2D Fourier transform in polar coordinates.

The outline of the paper is as follows. Section 2 states the proposed definition of the discrete 2D Fourier transform in polar coordinates. The motivation of this definition and the transform rules (multiplication, convolution, shift etc) are given in the first part of this two-part paper. The transform exists in its own right and manipulates discrete quantities that do not necessarily stem from sampling an underlying continuous quantity. Nevertheless, the motivation for the definition of the transform is based on an implied underlying discretization scheme. Section 3 introduces the implied underlying discretization scheme where we show the connection between discrete samples of the continuous functions and the discrete transform, should it be desirable to interpret the transform in this manner. Here, the connection between using the proposed 2D DFT and sampled vales of the continuous functions is explained. The proposed 2D DFT was motivated by a specific sampling scheme (section 3) which can be plotted and analyzed for 'grid coverage' - how much 
80 of the 2D plane is covered and at which density. Thus, Section 4 analyzes the proposed 81 discretization points and their implication on the sampling grid for density and coverage of the 82 grid. The insights gained from this section will be useful in interpreting the results of 83 approximating the continuous transform with the discrete transform. Section 5 introduces 84 numerical computation schemes whereby the interpretation of the proposed $2 \mathrm{D}$ transform as a 85 sequence of 1D DFT, 1D Discrete Hankel Transform (DHT) and 1D inverse DFT (IDFT) is 86 exploited. Section 6 then investigates the ability of the proposed 2D DFT to approximate the 87 continuous transform in terms of precision and accuracy. Three test functions for which closed88 form continuous transforms are known are analyzed. Finally, section 7 summarizes and 89 concludes the paper.

\section{0 \\ 2 Definition of the Discrete 2D Fourier Transform in Polar Coordinates}

91

92

93

94

95

96

97 98

100 101

102

The 2D-Discrete Fourier Transform in polar coordinates has been defined in the first part of this two-paper series as the discrete transform that takes the matrix (or double-subscripted series) $f_{p k}$ to the matrix (double-subscripted series) $F_{q l}$ such that $f_{p k} \rightarrow F_{q m}$ is given by

$$
F_{q m}=\mathrm{F}\left(f_{p k}\right)=\sum_{k=1}^{N_{1}-1} \sum_{p=-M}^{M} f_{p k} E_{q m ; p k}^{-}
$$

where $p, k, q, m, n, N_{1}$, and $N_{2}$ are integers such that $-M \leq n \leq M$, where $2 M+1=N_{2} \quad 1 \leq m, k, \leq N_{1}-1$ and $-M \leq p, q \leq M$. Unless otherwise stated, in the remainder of the paper it shall be assumed that $p, k, q, m, n, N_{1}$, and $N_{2}$ are within these stated ranges. Similarly, for the inverse transform we propose

$$
f_{p k}=\mathrm{F}^{-1}\left(F_{q m}\right)=\sum_{m=1}^{N_{1}-1} \sum_{q=-M}^{M} F_{q m} E_{q m ; p k}^{+}
$$

In equations (1) and (2), $E_{q m ; p k}^{ \pm}$are the kernels of the transformation. These can be chosen as the 'non-symmetric' form given by

$$
E_{q m ; p k}^{-}=\frac{1}{N_{2}} \sum_{n=-M}^{M} \frac{J_{n}\left(\frac{j_{n k} j_{n m}}{j_{n N_{1}}}\right)}{j_{n N_{1}}^{2} J_{n+1}^{2}\left(j_{n k}\right)} 2 i^{-n} e^{-i n \frac{2 \pi p}{N_{2}}} e^{+i n \frac{2 \pi q}{N_{2}}}
$$

$$
E_{q m ; p k}^{+}=\frac{1}{N_{2}} \sum_{n=-M}^{M} \frac{J_{n}\left(\frac{j_{n m} j_{n k}}{j_{n N_{1}}}\right)}{J_{n+1}^{2}\left(j_{n m}\right)} 2 i^{+n} e^{+i \frac{2 \pi n p}{N_{2}}} e^{-i \frac{2 \pi n q}{N_{2}}}
$$


103 Here, $J_{n}(z)$ is the $n$th order Bessel function of the first kind and $j_{n k}$ denotes the $k$ th zero of the

$104 n$th Bessel function. The subscript ( + or - ) indicated the sign on the $i^{ \pm}$and on the exponent

105 containing the $p$ variable; the $q$ variable exponent then takes the opposite sign. From a matrix point

106 of view, both $f_{p k}$ and $F_{q l}$ are $N_{2} \times\left(N_{1}-1\right)$ sized matrices. The form of the kernel in equation

107 (3) arises naturally from discretization of the continuous transform, but does not lead to the

108 expected Parseval relationship. A possible symmetric kernel is discussed in the first part of this

109 two-part paper and Parseval relationships are discussed further there [18].

\section{Discrete Transform to approximate the continuous transform}

111 In this section, relationships between discretely sampled values of the function and its continuous

112 2D FT are presented in the case of a space-limited or band-limited function. These relationships

113 were derived in the first part of the paper and are repeated here to demonstrate how they form the

114 basis for the using the discrete transform to approximate the continuous transform at specified

115 sampling points

\section{$1163.1 \quad$ Space-limited functions}

117 Consider a function in the space domain $f(r, \theta)$ which is space limited to $r \in[0, R]$. This implies

118 that the function is zero outside of the circle bounded by $r \in[0, R]$. An approximate relationship

119 between sampled values of the continuous function and sampled values of its continuous forward

120 2D transform $F(\rho, \psi)$ has been derived in the first part of the two-part paper as

$F\left(\frac{j_{q m}}{R}, \frac{2 \pi q}{N_{2}}\right) \approx 2 \pi R^{2} \sum_{k=1}^{N_{1}-1} \sum_{p=-M}^{M} f\left(\frac{j_{p k} R}{j_{p N_{1}}}, \frac{2 \pi p}{N_{2}}\right) \frac{1}{N_{2}} \sum_{n=-M}^{M} \frac{2 i^{-n} J_{n}\left(\frac{j_{n k} j_{n m}}{j_{n N_{1}}}\right)}{j_{n N_{1}}^{2} J_{n+1}^{2}\left(j_{n k}\right)} e^{-i \frac{2 \pi n p}{N_{2}}} e^{+i \frac{2 \pi n q}{N_{2}}}$

122 Similarly, an approximate relationship between sampled values of the continuous forward

123 transform $F(\rho, \psi)$ and sampled values of the continuous original function $f(r, \theta)$ was shown to

124 be given by

125

$$
f\left(\frac{j_{p k} R}{j_{p N_{1}}}, \frac{2 \pi p}{N_{2}}\right) \approx \frac{1}{2 \pi R^{2}} \sum_{m=1}^{N_{1}-1} \sum_{q=-M}^{M} F\left(\frac{j_{q m}}{R}, \frac{2 \pi q}{N_{2}}\right) \frac{1}{N_{2}} \sum_{n=-M}^{M} \frac{2 i^{n} J_{n}\left(\frac{j_{n m} j_{n k}}{j_{n N_{1}}}\right)}{J_{n+1}^{2}\left(j_{n m}\right)} e^{+i \frac{2 \pi n p}{N_{2}}} e^{-i \frac{2 \pi n q}{N_{2}}}
$$


126 In equations (4) and (5), $f(r, \theta)$ is the original function in $2 \mathrm{D}$ space and $F(\rho, \psi)$ is the $2 \mathrm{D}$ 127 Fourier transform of the function in polar coordinates.

128 To evaluate if the 2D DFT as proposed in equations (1) and (2) can be used to approximate 129 sampled values of $f(r, \theta)$ and $F(\rho, \psi)$, the process is as follows. For the forward transform, we 130 start with the continuous $f(r, \theta)$, evaluate it at the sampling points and then assign this value to $131 f_{p k}$ via

$$
f_{p k}=f\left(\frac{j_{p k} R}{j_{p N 1}}, \frac{2 \pi p}{N_{2}}\right)
$$

133 Then, $F_{q m}$ is calculated from the 2D DFT scaled by $2 \pi R^{2}$, equation (1), that is

$$
F_{q m}=2 \pi R^{2} \mathrm{~F}\left(f_{p k}\right)=2 \pi R^{2} \sum_{k=1}^{N_{1}-1} \sum_{p=-M}^{M} f_{p k} E_{q m ; p k}^{-} \quad \text { (7)The factor of } 2 \pi R^{2} \text { is necessary so }
$$

135 that the evaluation in (7) matches the expression in equation (4). To evaluate if the proposed 2D

136 DFT can be used to approximate the continuous transform, the question becomes how well $F_{q m}$

137 calculated from the 2D DFT in equation (7) approximates $F\left(\frac{j_{q m}}{R}, \frac{2 \pi q}{N_{2}}\right)$ - the values of the

138 continuous 2D Fourier transform evaluated on the sampling grid.

139 To evaluate the inverse 2D DFT, the process is similar. We start with the continuous

$140 F(\rho, \psi)$, evaluate it at the sampling points and assign this value to $F_{q m}$ via

$$
F_{q m}=F\left(\frac{j_{q m}}{R}, \frac{2 \pi q}{N_{2}}\right)
$$

142 Now, $f_{p k}$ is calculated from a scaled version of the inverse 2D DFT, equation (2) that is

$$
f_{p k}=\frac{1}{2 \pi R^{2}} \mathrm{~F}^{-1}\left(F_{q m}\right)=\frac{1}{2 \pi R^{2}} \sum_{m=1}^{N_{1}-1} \sum_{q=-M}^{M} F_{q m} E_{q m ; p k}^{+}
$$


144 To evaluate if the proposed transform can approximate the continuous transform, the question

145 becomes how well $f_{p k}$ calculated from equation (9) approximates $f\left(\frac{j_{p k} R}{j_{p N 1}}, \frac{2 \pi p}{N_{2}}\right)$ - the values of

146 the continuous function evaluated on the sampling grid.

\section{$147 \quad 3.2$ Band-limited functions}

148 The process for band-limited functions follows the same process as outlined in the previous 149 section, with the exception that the sampling points and scaling factors are slightly different as 150 they are now given in terms of the band limit rather than the space limit. Now consider functions 151 in the frequency domain $F(\rho, \psi)$ with an effective band limit $\rho \in\left[0, W_{\rho}\right]$. That is, we suppose

152 that the 2D Fourier transform $F(\rho, \psi)$ of $f(r, \theta)$ is band-limited, meaning that $F(\rho, \psi)$ is zero

153 for $\rho \geq W_{\rho}=2 \pi W$. The variable $W_{\rho}$ is written in this form since $W$ would typically be quoted

154 in units of $\mathrm{Hz}$ (cycles per second) if using temporal units or cycles per meter if using spatial units.

155 Therefore, the multiplication by $2 \pi$ ensures that the final units are in $s^{-1}$ or $m^{-1}$. The 156 approximate relationship between sampled values of the continuous 2D Fourier transform $157 F(\rho, \psi)$ and sampled values of the original continuous function $f(r, \theta)$ was derived in the first 158 part of the paper and is given by

$F\left(\frac{j_{q m} W_{\rho}}{j_{q N_{1}}}, \frac{2 \pi q}{N_{2}}\right) \approx \frac{2 \pi}{W_{\rho}^{2}} \sum_{k=1}^{N_{1}-1} \sum_{p=-M}^{M} f\left(\frac{j_{p k}}{W_{\rho}}, \frac{2 \pi p}{N_{2}}\right) \frac{1}{N_{2}} \sum_{n=-M}^{M} \frac{2 i^{-n} J_{n}\left(\frac{j_{n m} j_{n k}}{j_{n N_{1}}}\right)}{J_{n+1}^{2}\left(j_{n k}\right)} e^{-i \frac{2 \pi n p}{N_{2}}} e^{+i \frac{2 \pi n q}{N_{2}}}$

160 Similarly, the inverse relationship between sampled values of $F(\rho, \psi)$ and sampled values of $161 f(r, \theta)$ was shown

to

be

given

by

162

$$
f\left(\frac{j_{p k}}{W_{\rho}}, \frac{2 \pi p}{N_{2}}\right) \approx \frac{W_{\rho}^{2}}{2 \pi} \sum_{m=1}^{N_{1}-1} \sum_{q=-M}^{M} F\left(\frac{j_{q m} W_{\rho}}{j_{q N_{1}}}, \frac{2 \pi q}{N_{2}}\right) \frac{1}{N_{2}} \sum_{n=-M}^{M} \frac{2 i^{n} J_{n}\left(\frac{j_{n k} j_{n m}}{j_{n N_{1}}}\right)}{j_{n N_{1}}^{2} J_{n+1}^{2}\left(j_{n m}\right)} e^{-i \frac{2 \pi n q}{N_{2}}} e^{+i \frac{2 \pi n p}{N_{2}}}
$$

163 The relationships in equations (10) and (11) give relationships between the sampled values of the 164 original function and sampled values of its 2D Fourier transform. 

specific) sampling points and assign this value to $f_{p k}$ via

$$
f_{p k}=f\left(\frac{j_{p k}}{W_{\rho}}, \frac{2 \pi p}{N_{2}}\right)
$$

168 Then, $F_{q m}$ is calculated from the discrete transform scaled by $\frac{2 \pi}{W_{\rho}^{2}}$, equation (1), that is

$$
F_{q m}=\frac{2 \pi}{W_{\rho}^{2}} \mathrm{~F}\left(f_{p k}\right)=\frac{2 \pi}{W_{\rho}^{2}} \sum_{k=1}^{N_{1}-1} \sum_{p=-M}^{M} f_{p k} E_{q m ; p k}^{-}
$$

170 To evaluate if the proposed 2D DFT can be used to approximate the continuous transform, the

171 question is how well $F_{q m}$ calculated from equation (13) approximates $F\left(\frac{j_{q m} W_{\rho}}{j_{q N_{1}}}, \frac{2 \pi q}{N_{2}}\right)$, which are

172 the values of the continuous 2D Fourier transform, evaluated on the sampling grid. The evaluation

173 of the inverse transform for the band-limited function proceeds similarly by comparing values

174 obtained from the inverse 2D DFT to the values obtained by sampling the continuous function 175 directly.

176 The relationships given by equation (4),(5),(10) and (11), were the motivating definition of 177 a 2D Discrete Fourier transform in polar coordinates, defined in the first part of this two-part paper.

178 In the context of this second part of the two-part paper, they are also the relationships that permit

179 the use of the discrete transform to approximate the continuous transform at the specified sampling 180 points. They are also the relationships that permit the examination of whether the discrete quantities $f_{p k}$ and $F_{q m}$ calculated via the proposed 2D DFT are in fact reasonable approximations

182 to the sampled values of the continuous functions, as stated in the objectives of the paper.

\section{Discretization Points and Sampling Grid}

184 The transforms defined in equations (1) and (2) can be applied to any matrix $f_{p k}$ to yield its forward 185 transform $F_{q m}$, which can then be transformed backwards by using the inverse transform. However, 186 if these same discrete transforms are to be used for the purpose of approximating a continuous 2D 187 Fourier transform, then these transforms need to be applied to the specific sampled values of the 188 continuous functions in both space and frequency domains, as shown in equations (6), (8) and (12). 
189 The relationships in (4)and (10) define the sampling points that need to be used and it is noted that 190 the points are defined differently based on whether we start with the assumption of a space or band 191 limited function. These specific sampling points imply a specific sampling grid for the function.

192 In this section, the sampling grid (its coverage and density in 2D) is analyzed.

1934.1 Sampling points

194 For a space-limited function, we assume that the original function of interest is defined over 195 continuous $(r, \theta)$ space where $0 \leq r \leq R$ and $0 \leq \theta \leq 2 \pi$. The discrete sampling spaces used for 196 radial and angular sampling points in regular $\dot{r}$ space $(r, \theta)$ and $\stackrel{\prime}{\omega}$ frequency $(\rho, \psi)$ space are 197 defined as

$$
r_{p k}=\frac{j_{p k} R}{j_{p N_{1}}} \quad \theta_{p}=\frac{p 2 \pi}{N_{2}}
$$

199 and

$$
\rho_{q m}=\frac{j_{q m}}{R} \quad \psi_{q}=\frac{q 2 \pi}{N_{2}}
$$

201

202

203

204

205

206

For a band limited function, the function is assumed band-limited to $0 \leq \rho \leq W_{\rho}, 0 \leq \psi \leq 2 \pi$. The sampling space used for radial and angular sampling points in regular $\stackrel{1}{\omega}$ frequency space $(\rho, \psi)$ and $r$ space $(r, \theta)$ for a bandlimited function is defined as

$$
r_{p k}=\frac{j_{p k}}{W_{\rho}} \quad \theta_{p}=\frac{p 2 \pi}{N_{2}}
$$

and

$$
\rho_{q m}=\frac{j_{q m} W_{\rho}}{j_{q N_{1}}} \quad \psi_{q}=\frac{q 2 \pi}{N_{2}}
$$

Clearly, the density of the sampling points depends on the numbers of points chosen, that is on $N_{1}$ and $N_{2}$. Also clear is the fact that the grid is not equispaced in the radial variable. The sampling grid for a space-limited function are plotted below to enable visualization. In the first instance, the polar grids are plotted for the case $R=1, N_{1}=16$ and $N_{2}=15$. These are shown in space ( $r$ space) and frequency ( $\boldsymbol{\rho}$ space) in Figures 1 and 2 respectively. It should be noted that although we refer the grids in this paper as polar grids, they are not true polar grids in the sense of equispaced sampling in the radial and angular coordinates.

Clearly, the grids in Figure 1 and Figure 2 are fairly sparse, but the low values of $N_{2}$ and $N_{1}$ have been chosen so that the structure of the sampling points can be easily seen. It can be observed that there is a hole at the center area in both domains which is caused by the special sampling points. For higher values of the $N_{2}$ and $N_{1}$, the grid becomes fairly dense, obtaining 
218 good coverage of both spaces, but details are harder to observe. To demonstrate, the polar grids 219 are plotted for the case $R=1, N_{1}=96$ and $N_{2}=95$. These are shown in Figures 3 and 4 220 respectively.

221 From Figure 3 and Figure 4, by choosing higher values of $N_{1}$ and $N_{2}$, the sampling grid becomes denser, however there is still a gap in the center area. The sampling grids for band-limited functions are not plotted here since the sample grid for a band-limited function has the same shape as with space limited function but the domains are reversed.

225

226

227

228

229

230

231

232

233

234

235

236

237

238

239

240

241

242

243

244

245

246

\subsection{Sample Grid Analysis}

From part I of the paper, it was shown that the 2D-Fourier Transform can be interpreted as a Discrete Fourier Transform in the angular direction, a Discrete Hankel Transform in the radial direction and then an inverse Discrete Fourier Transform in the angular direction. Hence, the sample size in the angular direction could have been decided by the Nyquist sampling theorem [20], which states that

$$
f_{s}>2 f_{\max }
$$

where $f_{s}$ is the sample frequency and $f_{\max }$ is the highest frequency or band limit.

In the radial direction, the necessary relationship for the Discrete Hankel Transform is given by [21]

$$
W_{\rho} R=j_{n N_{1}}
$$

where $W_{\rho}$ is the effective band-limit, $R$ is the effective space limit and $j_{n N}$ is the $N^{\text {th }}$ zero of $J_{n}(r)$ . For the 2D Fourier Transform, since $-M \leq p \leq M$, the order of the Bessel zero ranges from $-M$ to $M$, the required relationship becomes

$$
\min \left(j_{p N_{1}}\right) \geq W_{\rho} R
$$

The relationships $j_{n N}=j_{-n N}$ and $j_{0 N_{1}}<j_{ \pm 1 N_{1}}<j_{ \pm 2 N_{1}}<\ldots<j_{ \pm M N_{1}}$ are valid [22], hence equation (20) can be written as

$$
j_{0 N_{1}} \geq W_{\rho} R
$$

It is pointed out in [23], [24] that the zeros of $J_{n}(z)$ are almost evenly spaced at intervals of $\pi$ and that the spacing becomes exactly $\pi$ in the limit as $z \rightarrow \infty$. The reader unfamiliar with Bessel functions is directed to references [19], [22]. In fact, it is shown in [10] that a simple asymptotic form for the Bessel function is given by

$$
J_{n}(z) \approx \sqrt{\frac{2}{\pi z}} \cos \left[z-\left(n+\frac{1}{2}\right) \frac{\pi}{2}\right]
$$

Therefore, an approximation to the Bessel zero, $j_{n k}$ is given by

Peer) Comput. Sci. reviewing PDF | (CS-2019:07:39130:2:0:NEW 30 Dec 2019) 


$$
j_{n k} \approx\left(2 k+n-\frac{1}{2}\right) \frac{\pi}{2}
$$

250

251

252

253

254

255

256

257

258

259

260

261

262

263

264

265

266

267

268

269

270

271

272

273

274

275

276

277

Hence, equation (21) can be written to choose $N_{1}$ approximately as

$$
\begin{aligned}
& N_{1} \pi \geq W_{\rho} R=2 \pi W R \\
& \Rightarrow N_{1} \geq 2 W R
\end{aligned}
$$

where the reader is reminded that the units of $W$ is $m^{-1}$ (the space equivalent of $\mathrm{Hz}$ ). $N_{1} / R$ is the spatial sampling frequency and we see that equation (24) effectively makes the same statement as equation (18), as it should.

Intuitively, more sample points lead to more information captured, which gives an expectation that increasing $N_{1}$ or $N_{2}$ individually will give a better sampling grid coverage. However, it can be seen from Figure 1 to Figure 4 that there is a gap in the center of the sample grid. From equation (14) and (15), the area of the gap in the center is related to the ranges of $p$ and $k$, that is $N_{2}$ and $N_{1}$. In the sections below, it is assumed that the sampling theorems are already satisfied (that is, an appropriate space and band limit is selected) and the relationship between $N_{2}$, $N_{1}$ and the size of the gap will be discussed.

\subsubsection{Space-limited function}

In this section, it is assumed that the function is a space limited function, defined in $r \in[0, R]$. The sampling points are defined as equation (14) in the space domain and (15) in the frequency domain. In the following, a relationship between $N_{2}, N_{1}$ and the area of the gap in both domains is discussed.

\subsubsection{Sample grid in the space domain}

In the space domain, the effective limit in the space domain, $R$, is fixed. To analyze how the values of $N_{2}$ and $N_{1}$ affect the coverage of the grid in space domain, consider the following definition of 'grid coverage'

$$
A_{r}=\frac{\pi R^{2}-\pi \bar{r}^{2}}{\pi R^{2}} \cdot 100
$$

where $\bar{r}$ denotes the average radius of the gap (the hole in the middle of the grid). $A_{r}$ as defined in equation (25) is a measure of the 'grid coverage' since it gives a percentage of how much of the original space limited domain area is captured by the discrete grid. For example, if the average radius of the center gap is zero, then $A_{r}$ would be $100 \%$, that is, complete coverage. Based on the observation of Figure 1 and Figure 3, the relationship $r_{01}<r_{ \pm 11}<r_{ \pm 21}<r_{ \pm M 1}$ is valid. Therefore, from equation (14), the average radius of the gap is given by 


$$
\bar{r}=\frac{\left(r_{01}+r_{M 1}\right)}{2}=\frac{1}{2}\left(\frac{j_{01}}{j_{0 N_{1}}} R+\frac{j_{M 1}}{j_{M N_{1}}} R\right)
$$

279

280

281

282

283

284

285

286

287

288

289

290

292

293

294

295

296

297

298

299

300

301

302

Hence, equation (25) for grid coverage can be written as

$$
A_{r}=\left[1-\frac{1}{4}\left(\frac{j_{01}}{j_{0 N_{1}}}+\frac{j_{M 1}}{j_{M N_{1}}}\right) 2\right] \cdot 100
$$

Table 1 shows the different values of grid coverage $A_{r}$ as the values of $N_{1}$ and $N_{2}$ are changed.

From Table 1, it can be seen that increasing $N_{1}$ (sample size in the radial direction) tends to increase the grid coverage. Since the effective space limit $R$ is fixed, from equation (21) it follows that increasing $N_{1}$ actually increases the effective band limit. However, increasing $N_{2}$ (sample size in angular direction) will result in a bigger gap in the center of the grid, which then decreases the coverage.

\subsubsection{Sample grid in the frequency domain}

Similarly, coverage of the grid in the frequency domain is defined as

$$
A_{\rho}=\frac{\pi W_{\rho}^{2}-\pi \bar{\rho}^{2}}{\pi W_{\rho}^{2}} \cdot 100
$$

where $\bar{\rho}$ denotes the average radius of the gap. Since

$$
\bar{\rho}=\frac{\left(\rho_{01}+\rho_{M 1}\right)}{2}=\frac{\left(j_{01}+j_{M 1}\right)}{2 R}
$$

Then, it follows that equation (28) for frequency domain grid coverage can be written as

$$
A_{\rho}=\left[1-\frac{\left(j_{01}+j_{M 1}\right)^{2}}{4 R^{2} W_{\rho}^{2}}\right] \cdot 100 \%
$$

From equation (30), it can be observed that the sample grid coverage in the frequency domain is affected by $R, W_{\rho}$ and $M$. Since $N_{2}=2 M+1$, in order to get a better grid coverage with a fixed $W_{\rho}, R$ and $N_{2}$ can be adjusted. Table 2 shows the grid coverage $A_{\rho}$ for different values of $R$ and $N_{2}$.

From Table 2, the conclusion for the frequency domain is that when the effective band limit is fixed, increasing $R$ (effective space limit) tends to increase the coverage in the frequency domain, while increasing $N_{2}$ (sample size in the angular direction) decreases the coverage. However, from equation (21) it should be noted that to satisfy the sampling theorem, increasing $R$ with fixed $W_{\rho}$ requires an increase in $N_{1}$ at the same time. 
303

304

305

306

307

308

309

310

311

312

313

\subsubsection{Band-limited function}

In this section, we suppose that the function is an effectively band limited function, defined on $\rho \in\left[0, W_{p}\right]$. The sampling points are defined as in equation (16) in the space domain and as in

(17) in the frequency domain. In this subsection, the relationship between $N_{2}, N_{1}$ and the area of the gap in both domains is discussed.

\subsubsection{Sampling Grid in the space domain}

The same definition of grid coverage in the space domain will be used as in equation (25). Since the sampling points of a band-limited function are given by equations (16) and(17), the average radius of the gap can be defined as

$$
\bar{r}=\frac{\left(r_{01}+r_{M 1}\right)}{2}=\frac{1}{2}\left(\frac{j_{01}}{W_{\rho}}+\frac{j_{M 1}}{W_{\rho}}\right)
$$

Therefore, the coverage of the grid in space domain can be written as

$$
A_{r}=\left[1-\frac{\left(j_{01}+j_{M 1}\right)^{2}}{4 W_{\rho}^{2} R^{2}}\right] \cdot 100
$$

It can be observed that the grid coverage in the space domain of a band-limited function is the same as the grid coverage in the frequency domain of space limited function.

\subsubsection{Sample Grid in frequency domain}

The coverage of the grid in the frequency domain of a band limited function is defined by equation (28). With sampling points defined in equation (17), the average radius of the gap can be defined as

$$
\bar{\rho}=\frac{\left(\rho_{01}+\rho_{M 1}\right)}{2}=\frac{1}{2}\left(\frac{j_{01}}{j_{0 N_{1}}} W_{\rho}+\frac{j_{M 1}}{j_{M N_{1}}} W_{\rho}\right)
$$

The coverage of the grid in frequency domain can be written as

$$
A_{\rho}=\left[1-\frac{1}{4}\left(\frac{j_{01}}{j_{0 N_{1}}}+\frac{j_{M 1}}{j_{M N_{1}}}\right)^{2}\right] \cdot 100
$$

It can be observed that the grid coverage in the frequency domain of a band-limited function is the same as the grid coverage in the space domain of a space limited function.

\subsection{Conclusion}

Based on the discussion above, the following conclusions can be made:

1. Increasing $N_{2}$ (angular direction) tends to decrease the sampling grid coverage in both domains. Increasing $N_{1}$ (radial direction) tends to increase the sampling coverage in the space domain for a space-limited function and in the frequency domain for a frequencylimited function. So, if a signal changes sharply in the angular direction such that large 
332

333

334

335

336

337

338

339

340

341

342

\section{3}

344

345

346

347

348

349

350

351

352

353

354

355

356

\subsection{Forward transform}

The values $f_{p k}$ can be considered as the entries in a matrix. To transform $f_{p k} \rightarrow F_{q m}$, the operation is performed as a sequence of steps which are a 1D DFT (column-wise), followed by a scaled 1D DHT (row-wise), finally followed by a 1D inverse DFT (column-wise). The reader is reminded that the range of indices is given by $m, k=1 . . N_{1}-1$ and $n, p, q=-M . . M$, where $2 M+1=N_{2}$. These steps can be summarized succinctly by rewriting equation (1) as

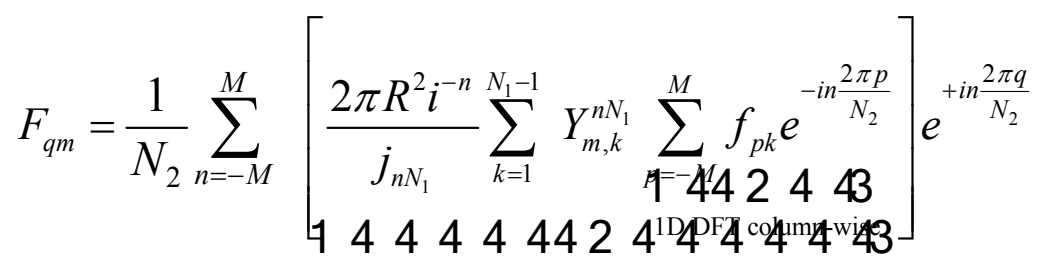

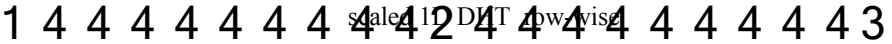

inverse 1D DFT column-wise

where the DHT is defined in [21] via the transformation matrix 


$$
Y_{m, k}^{n N_{1}}=\frac{2}{j_{n N_{1}} J_{n+1}^{2}\left(j_{n k}\right)} J_{n}\left(\frac{j_{n m} j_{n k}}{j_{n N_{1}}}\right) \quad 1 \leq m, k \leq N_{1}-1
$$

359 Matlab code for the DHT is described in [25]. The inverse 2D DFT can be similarly interpreted, as shown in section 5.2.

\section{$361 \quad 5.2$ Inverse Transform}

362 The steps of the inverse 2D DFT are the reverse of the steps outlined above for the forward 2D 363 DFT. For $p=-M . . M$ and $k=1 . . N_{1}-1$, equation (2) this can be expressed as

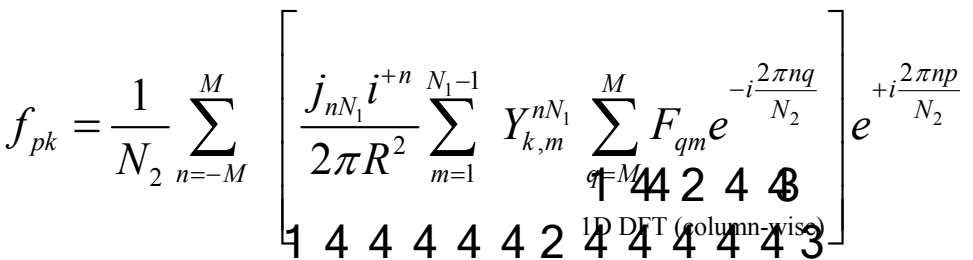

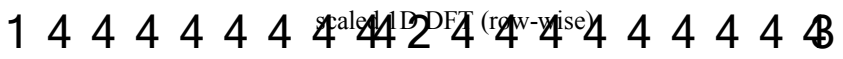

365

366

367

368

369

370

371

372

373

374

375

376

377

378

379

380

This parallels the steps taken for the continuous case, with each continuous operation (Fourier series, Hankel transform) replaced by its discrete counterpart (DFT, DHT).

Therefore, for both forward and inverse 2D-DFT, the sequence of operations is a DFT of each column of the starting matrix, followed by a DHT of each row, a term-by-term scaling, followed by an IDFT of each column. This is a significant computational improvement because by interpreting the transform this way, the Fast Fourier Transform (FFT) can be used, which reduces the computational time quite significantly in comparison with a direct implementation of the summation definitions in equations (1) and (2).

\subsection{Interpretation of the sampled forward transform in Matlab terms}

To use the built-in Matlab function $f f t$, a few operations are required. First, we define Matlabfriendly indices $\quad p^{\prime}=p+(M+1) \quad$ and $\quad n^{\prime}=n+(M+1) \quad$ so that $p, n=-M . . M$ become $p^{\prime}, n^{\prime}=1 . .2 M+1=1 . . N_{2}\left(\right.$ since $\left.2 M+1=N_{2}\right)$. That is, the primed variables range from $1 . .2 M$ rather than $-M . . M$. Hence, if the matrix $\mathbf{f}$ with entries $f_{p^{\prime} k}$ is defined, where $p^{\prime}=1 . . N_{2}, k=1 . . N_{1}-1$, then the first step in (35) which is a column-wise DFT can be written as the Matlab-defined DFT as

$$
\bar{f}_{n^{\prime} k}=\sum_{p^{\prime}=1}^{N_{2}} f_{p k} e^{\frac{-2 \pi i\left(p^{\prime}-1-M\right)\left(n^{\prime}-1-M\right)}{N_{2}}}
$$


381 The overbar denotes a discrete Fourier transform. The definition of DFT in Matlab is actually 382 given by the relationship

$$
\bar{f}_{n^{\prime} k}=\sum_{p^{\prime}=1}^{N_{2}} f_{p^{\prime} k} e^{\frac{-2 \pi i\left(p^{\prime}-1\right)\left(n^{\prime}-1\right)}{N_{2}}}
$$

384 Since the relationship $\sum_{p^{\prime}=1}^{N_{2}} f_{p^{\prime} k} e^{\frac{-2 \pi i\left(p^{\prime}-1\right)\left(n^{\prime}-1-M\right)}{N 2}}=\sum_{p^{\prime}=1}^{N_{2}} f_{p k} e^{\frac{-2 \pi i\left(p^{\prime}-1-M\right)\left(n^{\prime}-1-M\right)}{N 2}}$ is valid, we can sample the 385 original function to obtain the discrete $f_{p k}$ values, put them in the matrix $f_{p^{\prime} k}$ then shift the matrix $386 f_{p^{\prime} k}$ by $M+1$ along the column direction. In Matlab, the function $\operatorname{circshift}(A, K, \operatorname{dim})$ can be 387 used, which circularly shifts the values in array $A$ by $K$ positions along dimension dim. Inputs $388 K$ and $\operatorname{dim}$ must be scalars. Specifically, $\operatorname{dim}=1$ indicates the columns of matrix $A$ and $\operatorname{dim}=2$ 389 indicates the rows of matrix A. Hence, equation (38) can be written as

$$
\bar{f}_{n^{\prime} k}=f f t\left(\operatorname{circshift}\left(f_{p^{\prime} k}, M+1,1\right), N_{2}, 1\right)
$$

391 In matrix operations, this is equivalent to stating that each column of $f_{p^{\prime} k}$ is DFT'ed to yield $\bar{f}_{n^{\prime} k}$. The second step in equation (35) is a discrete Hankel transform of order $n$, transforming

$393 \bar{f}_{n^{\prime} k} \rightarrow \hat{\bar{f}}_{n^{\prime} l}$ so that the $k$ subscript is Hankel transformed to the $l$ subscript. The overhat denotes 394 a discrete Hankel transform. In order to relate the order $n$ to the index $n^{\prime}$, we need to shift $\bar{f}_{n^{\prime} k}$

395 by $-(M+1)$ along column direction so that the order ranges from $-M$ to $M$.

$$
\hat{\bar{f}}_{n^{\prime} l}=\sum_{k=1}^{N_{1}-1} \frac{2 J_{n}\left(\frac{j_{n k} j_{n l}}{j_{n N_{1}}}\right)}{j_{n N_{1}} J_{n+1}^{2}\left(j_{n k}\right)} \operatorname{circshift}\left(\bar{f}_{n^{\prime} k},-(M+1), 1\right) \quad\left\{\begin{array}{c}
\text { for } n^{\prime}=1 . . N_{2}, l=1 . . N_{1}-1 \\
\text { where } n=n^{\prime}-(M+1)
\end{array}\right.
$$

397 By using the Hankel transform matrix defined in [21], equation (41) can be rewritten as

$$
\hat{\bar{f}}_{n^{\prime} l}=\operatorname{circshift}\left(\bar{f}_{n^{\prime} k},-(M+1), 1\right)\left(Y_{l, k}^{n N_{1}}\right)^{T} \quad\left\{\begin{array}{c}
\text { for } n^{\prime}=1 . . N_{2}, l=1 . . N_{1}-1 \\
\text { where } n=n^{\prime}-M-1
\end{array}\right.
$$


399 In matrix operations, this states that each row of $\bar{f}_{n^{\prime} k}$ is DHT'ed to yield $\hat{\bar{f}}_{n^{\prime} l}$. These are now 400 scaled to give the Fourier coefficients of the 2D DFT $\hat{\bar{f}}_{n^{\prime} l} \rightarrow \bar{F}_{n^{\prime} l}$. In order to proceed to an inverse 401 DFT in the next step, it is necessary to shift the matrix by $M+1$ along the column direction after 402 scaling

$$
\bar{F}_{n^{\prime} l}=\operatorname{circshift}\left(\frac{2 \pi R^{2}}{j_{n N_{1}}} i^{-n} \hat{\bar{f}}_{n^{\prime} l}, M+1,1\right) \quad\left\{\begin{array}{c}
\text { for } n^{\prime}=1 . . N_{2}, l=1 . . N_{1}-1 \\
\text { where } n=n^{\prime}-(M+1)
\end{array}\right.
$$

404 This last step is a 1D IDFT for each column of $\bar{F}_{n^{\prime} l}$ to obtain $F_{q l}$. Using $2 M+1=N_{2}$, and $405 q^{\prime}=q+1+M$, this can be written as

406

$$
\begin{aligned}
F_{q^{\prime} l} & =\frac{1}{N_{2}} \sum_{n^{\prime}=1}^{N_{2}} \bar{F}_{n l} e^{+i\left(n^{\prime}-M-1\right) \frac{2 \pi\left(q^{\prime}-1-M\right)}{N_{2}}} \quad \text { for } q^{\prime}=1 . . N_{2}, l=1 . . N_{1}-1 \\
& =\frac{1}{N_{2}} \sum_{n^{\prime}=1}^{N_{2}} \bar{F}_{n^{\prime} l} e^{+i\left(n^{\prime}-1\right) \frac{2 \pi\left(q^{\prime}-1-M\right)}{N_{2}}} \\
& =\operatorname{circshift}\left(\operatorname{ifft}\left(\bar{F}_{n^{\prime} l}, N_{2}, 1\right),-(M+1), 1\right)
\end{aligned}
$$

\section{$408 \quad 5.4$ Interpretation of the sampled inverse transform in Matlab terms}

409 Similar to the forward transform, matlab-friendly indices $q^{\prime}=q+(M+1)$ and $n^{\prime}=n+(M+1)$ 410 are also defined. Hence, if the matrix $F$ with entries $F_{q^{\prime} l}$ is defined, where $411 q^{\prime}=1 . . N_{2}, l=1 . . N_{1}-1$, it then follows that the first 1D DFT step in equation (37) can be written 412 as the Matlab-defined DFT as

413

$$
\bar{F}_{n^{\prime} l}=\sum_{q^{\prime}=1}^{N_{2}} F_{q l} e^{-i\left(n^{\prime}-M-1\right) \frac{2 \pi\left(q^{\prime}-1-M\right)}{N_{2}}} \quad \text { for } n^{\prime}=1 . . N_{2}, l=1 . . N_{1}-1
$$

$$
=\sum_{q^{\prime}=1}^{N_{2}} F_{q^{\prime} l} e^{-i\left(n^{\prime}-M-1\right) \frac{2 \pi\left(q^{\prime}-1\right)}{N_{2}}}
$$


414 If the original function can be sampled as $F_{q l}$ and then put into matrix $F_{q^{\prime} l}$, then we need an 415 circshift operation. So equation (45) can be written as

416

$$
\bar{F}_{n^{\prime} l}=f f t\left(\operatorname{circshift}\left(F_{q^{\prime} l}, M+1,1\right), N_{2}, 1\right)
$$

417 Subsequently, a discrete Hankel transform of order $n$ is required, transforming $\bar{F}_{n^{\prime} l} \rightarrow \hat{\bar{F}}_{n^{\prime} l}$ so that 418 the $l$ subscript is Hankel transformed to the $k$ subscript. To achieve this, circshift is also needed 419 here.

$$
\hat{\bar{F}}_{n^{\prime} k}=\operatorname{circshift}\left(\bar{F}_{n^{\prime} l},-(M+1), 1\right)\left(Y_{k, l}^{n N_{1}}\right)^{T} \quad\left\{\begin{array}{c}
\text { for } n^{\prime}=1 . . N_{2}, l=1 . . N_{1}-1 \\
\text { where } n=n^{\prime}-M-1
\end{array}\right.
$$

421 This is followed by a scaling operation to obtain $\hat{\bar{F}}_{n^{\prime} k} \rightarrow \bar{f}_{n^{\prime} k}$ and then a $\operatorname{circshift}$ by $(M+1)$ so 422 that

$$
\bar{f}_{n^{\prime} k}=\operatorname{circshift}\left(\frac{j_{n N_{1}}}{2 \pi R^{2}} i^{+n} \hat{\bar{F}}_{n^{\prime} k},(M+1), 1\right) \quad\left\{\begin{array}{c}
\text { for } n^{\prime}=1 . . N_{2}, k=1 . . N_{1}-1 \\
\text { where } n=n^{\prime}-(M+1)
\end{array}\right.
$$

424 This last step is a 1D IDFT for each column of $\bar{f}_{n^{\prime} k}$ to get $f_{p^{\prime} k}$. Using $2 M+1=N_{2}$, and $425 p^{\prime}=p+1$, equation (37) can be written as

$$
\begin{aligned}
f_{p^{\prime} \mathrm{k}} & =\frac{1}{N_{2}} \sum_{n^{\prime}=1}^{N_{2}} \bar{f}_{n k} e^{+i\left(n^{\prime}-M-1\right) \frac{2 \pi\left(p^{\prime}-1-M\right)}{N_{2}}} \quad \text { for } p^{\prime}=1 . . N_{2}, k=1 . . N_{1}-1 \\
& =\frac{1}{N_{2}} \sum_{n^{\prime}=1}^{N_{2}} \bar{f}_{n^{\prime} k} e^{+i \frac{2 \pi\left(n^{\prime}-1\right)\left(p^{\prime}-1-M\right)}{N_{2}}} \\
& =\operatorname{circshift}\left(\operatorname{ifft}\left(\bar{f}_{n^{\prime} k}, N_{2}, 1\right),-(M+1), 1\right)
\end{aligned}
$$

427 In conclusion, in this section, by using the interpretation of the kernel as sequential DFT, DHT and 428 IDFT operations, Matlab (or similar software) built-in code can be used to efficiently implement 429 the 2D DFT algorithm in polar coordinates. 
4306 Numerical evaluation of the 2D DFT in polar coordinates to 431 approximate the continuous FT

432 In this section, the 2D discrete Fourier transform is evaluated for its ability to estimate the

433

434

435

436

437

438

441

442

443

444

445

446

447

448

449

450

451

452

453

454

455

456

457

458

459

460

461

462

463

464

465

466

continuous Fourier transform at the selected special sampling points in the spatial and frequency domains.

\subsection{Method for testing the Algorithm}

\subsubsection{Accuracy}

In order to test accuracy of the 2D-DFT and 2D-IDFT to calculate approximate the continuous counterpart, the dynamic error is proposed as a metric. The dynamic error is defined as [24]

$$
E(\mathrm{v})=20 \log _{10}\left[\frac{|C(\mathrm{v})-D(\mathrm{v})|}{\max |D(\mathrm{v})|}\right]
$$

where $C(\mathrm{v})$ is the continuous forward or inverse 2D-Fourier transform and $D(\mathrm{v})$ is the value obtained from the discrete counterpart. The dynamic error is defined as the ratio of the absolute error to the maximum amplitude of the discrete function, calculated on a log scale. Therefore, a large negative value represents an accurate discrete transform. The dynamic error is used instead of the percentage error in order to avoid division by zero.

\subsubsection{Precision}

The precision of the algorithm is an important evaluation criterion, which is tested by sequentially performing a pair of forward and inverse transforms and comparing the result to the original function. High precision indicates that numerical evaluation of the transform does not add much error. An average of the absolute error between the original function and the calculated counterpart at each sample point is used to measure the precision. It is given by

$$
\varepsilon=\frac{1}{\left(N_{1}-1\right) \cdot N_{2}} \sum_{n=1}^{\left(N_{1}-1\right) \cdot N_{2}}\left|f-f^{*}\right|
$$

where $f$ is the original function and $f^{*}$ is the value obtained after sequentially performing a forward and then inverse transform. An ideal precision would result in the absolute error being zero.

\subsection{Test functions}

In this section, three test functions are chosen to evaluate the ability of the discrete transform to approximate the continuous counterpart. The first test case is the circularly symmetric Gaussian function. Given that it is circularly symmetric and that the Gaussian is continuous and smooth, the proposed DFT is expected to perform well. The second test case is "Four-term sinusoid and Sinc" function, which is not symmetric in the angular direction and suffers a discontinuity in the radial direction. The third test function presents a more challenging test function, the "Four-term sinusoid and Modified exponential" function. In this case, the test function is not circularly symmetric and it explodes at the origin (approaches infinity at the origin). Given that as shown above, the sampling grid cannot cover the area around the origin very well, a function that explodes at the origin should give more error and should provide a reasonable test case for evaluating the performance of the discrete transform. The test functions are chosen to test specific aspects of the 
467 performance of the discrete transform but also because a closed-form expression for both the 468 function and its transform are available. This then allows a numerical evaluation of the error 469 between the quantities computed with the 2D DFT and the quantities obtained by evaluating 470 (sampling) the continuous (forward or inverse) transform at the grid points.

$471 \quad 6.2 .1$ Gaussian

472 The first function chosen for evaluation is a circular symmetric function which is Gaussian in the radial direction. Specifically, the function in the space domain is given by

$$
f(r, \theta)=e^{-a^{2} r^{2}}
$$

475

476

477

478

479

480

481

482

483

484

485

486

487

488

489

490

491

492

493

494

495

496

497

498

499

500

501

where $a$ is some real constant. Since the function is circularly symmetric, the 2D-DFT is a zerothorder Hankel Transform [26] and is given by

$$
F(\rho, \psi)=\frac{\pi}{a^{2}} e^{\frac{-\rho^{2}}{4 a^{2}}}
$$

The graphs for the original function and its continuous 2D-DFT (which is also a Gaussian) are plotted with $a=1$ and shown in Figure 5. From Figure 5, the function is circular symmetric and fairly smooth in the radial direction. Moreover, the function can be considered as either an effectively space-limited function or an effectively band-limited function. For the purposes of testing it, it shall be considered as a space-limited function and equations (14) and (15) will be used to proceed with the forward and inverse transform in sequence.

To perform the transform, the following variables need to be chosen: $N_{2}, R$ and $N_{1}$. In the angular direction, since the function in the spatial domain is circularly symmetric, $N_{2}$ can be chosen to be small. Thus, $N_{2}=15$ is chosen.

In the radial direction, from plotting the function, it can be seen that the effective space limit can be taken to be $R=5$ and the effective band limit can be taken to be $W_{\rho}=10$. From equation (21), $j_{0 N_{1}} \geq R \cdot W_{\rho}=50$. Therefore, $N_{1}=17$ is chosen (we could also have obtained a rough estimate of $N_{1}$ from equation (24)). However, most of the energy of the function in both the space and frequency domains is located in the center near the origin. Based on the discussion in Section 4.3, relatively large values of $R$ and $W_{\rho}$ are needed. The effective space limit $R=40$ and effective band-limit $W_{p}=30$ are thus chosen, which gives $j_{0 N_{1}} \geq R \cdot W_{\rho}=1200$. Therefore $N_{1}=383$ is chosen in order to satisfy this constraint. Both cases discussed here $\left(N_{1}=17\right.$ and $N_{1}=383$ ) are tested in following.

\subsubsection{Forward Transform}

Test results with $R=5, N_{1}=17$ are shown in Figure 6 and Figure 7. Figure 6 shows the sampled continuous forward transform and the discrete forward transform. Figure 7 shows the error between the sampled values of the continuous transform and the discretely calculated values.

From Figure 7, it can be observed that the error gets bigger at the center, which is as expected because the sampling grid shows that the sampling points can never attain the origin. The 
502 maximum value of the error is $E_{\max }=-0.9115 \mathrm{~dB}$ and this occurs at the center. The average error 503 is $E_{\text {avg. }}=-30.4446 \mathrm{~dB}$.

504 Error test results with $R=40, N_{1}=383$ are shown in Figure 8. Similar to the previous

505

506

507

508

509

510 case, the error gets larger at the center, as expected. However, the maximum value of the error is $E_{\max }=-8.3842 \mathrm{~dB}$ and this occurs at the center. The average value of the error is $E_{\text {avg. }}=-63.8031 \mathrm{~dB}$. Clearly, the test with $R=40, N_{1}=383$ gives a better approximation, which verifies the discussion in Section 4.3.

With $R=40$, Table 3 shows the errors (max and average error) with respect to different value of $N_{1}$ and $N_{2}$. The trends as functions of $N_{1}$ and $N_{2}$ are shown as plots in Figure 9 and Figure 10.

From Figure 9, it can be seen that when $N_{1}$ individually $\left(N_{2}\right.$ is fixed at $N_{2}=15$ ) is less than the minimum of 383 obtained from the sampling theorem, increasing $N_{1}$ will lead to smaller errors, as expected. When $N_{1}$ is bigger than the sampling-theorem threshold of 383 , increasing $N_{1}$ still decreases the error which verifies the discussion about sample grid coverage in Section 4.3. Increasing $N_{1}$ tends to increase the sample grid coverage and capture more information at the center area and thus leads to smaller errors.

From Figure 10, increasing $N_{2}$ alone (that is, without a corresponding increase in $N_{1}$ ) leads to larger errors, both Error ${ }_{\max }$ and Error ${ }_{\text {average }}$. Although at first counterintuitive, this result is actually reasonable because the function is radially symmetric which implies that $N_{2}=1$ should be sufficient based on the sampling theorem for the angular direction. Therefore, increasing $N_{2}$ will not lead to a better approximation. Moreover, from the discussion of the sample grid coverage in Section 4.3, the sampling grid coverage in both domains gets worse when $N_{2}$ gets bigger because more information from the center is lost. This problem can be solved by increasing $N_{1}$ at the same time, but it could be computationally time consuming. Therefore, choosing $N_{2}$ properly is very important from the standpoint of accuracy and computational efficiency.

\subsubsection{Inverse Transform}

Test results for the inverse transform with $R=5, N_{1}=17$ are shown in Figure 11 and Figure 12. Figure 11 shows the sampled continuous inverse transform and discrete inverse transform and Figure 12 shows the error between the sampled continuous and discretely calculated values.

Similar to the case for the forward transform, the error gets larger at the center, which is as expected because the sampling grid shows that the sampling points never attain the center. The maximum value of the error is $E_{\max }=3.1954 d B$ and this occurs at the center. The average of the error is $E_{\text {avg. }}=-25.7799 \mathrm{~dB}$. 
535

536

537

538

539

540

541

542

543

544

545

546

547

548

549

550

551

552

553

554

555

556

557

558

559

560

Error test results for the inverse transform with $R=40, N_{1}=383$ are shown in Figure 13 .

In this case, the maximum value of the error is $E_{\max }=-12.2602 \mathrm{~dB}$ and this occurs at the center. The average of the error is $E_{\text {avg. }}=-98.0316 \mathrm{~dB}$. Table 4 shows the errors with respect to different value of $N_{1}$ and $N_{2}$, from which Figure 14 and Figure 15 demonstrate the trend.

From Figure 15 it can be observed that increasing $N_{1}$ tends to improve the result but not significantly. This could be explained by the discussion for $R=40, N_{1}=383$ that with fixed $R$ and $W_{\rho}$, increasing $N_{1}$ will not allow the sampling grid in the frequency domain to get any closer to the origin to capture more information. From Figure 15, increasing $N_{2}$ (with fixed $N_{1}=383$ ) leads to a worse approximation which verifies the discussion for $R=40, N_{1}=383$.

Performing sequential 2D-DFT and 2D-IDFT results in $\varepsilon=4.1656 \times e^{-17}$ where $\varepsilon$ is calculated with equation (51). Therefore, performing sequential forward and inverse transforms does not add much error.

6.2.2 Four-term sinusoid \& Sinc Function

The second function chosen for evaluation is given by

$$
f(r, \theta)=\frac{\sin (a r)}{a r}[3 \sin (\theta)+\sin (3 \theta)+4 \cos (10 \theta)+12 \sin (15 \theta)]
$$

which is a sinc function in the radial direction and a four-term sinusoid in the angular direction. The graphs for the original function and the magnitude of its continuous 2D-FT with $a=5$ are shown in Figure 16. From Figure 16, the function can be considered as a band-limited function. Therefore equation (16) and (17) were used to implement the forward and inverse transform.

The continuous 2D-FT can be calculated from [27]

$$
F(\rho, \psi)=\sum_{n=-\infty}^{\infty} 2 \pi i^{-n} e^{i n \psi} \int_{0}^{\infty} f_{n}(r) J_{n}(\rho r) r d r
$$

where $f_{n}(r)$ is the Fourier series of $f(r, \theta)$ and can be written as

$$
f_{n}(r)=\frac{1}{2 \pi} \int_{-\pi}^{\pi} f(r, \theta) e^{-i n \theta} d \theta
$$

From the sampling theorem for the angular direction, the highest angular frequency in equation (54) results in $N_{2}$ being at least 31 required to reconstruct the signal. Therefore, at least 31 terms are required to calculate the continuous $2 \mathrm{D}-\mathrm{FT}$, which can be written as 


$$
F(\rho, \psi)=\left\{\begin{array}{l}
\frac{8 \pi \cos (10 \psi) \rho^{10}}{a \sqrt{a^{2}-\rho^{2}}\left(a+\sqrt{a^{2}-\rho^{2}}\right)^{10}}, \rho<a \\
-\frac{6 \pi i \sin (\psi)}{a \rho \sqrt{\rho^{2}+a^{2}}}+\frac{2 \pi i \sin \left(3 \arcsin \left(\frac{a}{\rho}\right)\right) \sin (3 \psi)}{\sqrt{\rho^{2}+a^{2}}}-\frac{8 \pi \sin \left(10 \arcsin \left(\frac{a}{\rho}\right)\right) \cos (10 \psi)}{\sqrt{\rho^{2}+a^{2}}} \\
+\frac{24 \pi i \sin \left(15 \arcsin \left(\frac{a}{\rho}\right)\right) \sin (15 \psi)}{\sqrt{\rho^{2}+a^{2}}}, \rho>a
\end{array}\right.
$$

562

563

564

565

566

567

568

569

570

571

572

573

574

575

576

577

578

579

580

581

582

583

584

585

586

587

In the angular direction, the highest frequency term in the function in the space domain is $12 \sin (15 \theta)$. From the sampling theorem, the sampling frequency should be at least twice that of the highest frequency present in the signal. Thus, $N_{2}=41$ is chosen in order to go a little past the minimum requirement of 31 . In the radial direction, from the graphs of the original function and its 2D-FT, it can be assumed that $f(r, \theta)$ is space-limited at $R=15$ and band-limited at $W_{\rho}=30$. However, since most of the energy in the space domain is located at the origin, a relatively large band limit should be chosen based on the discussion in Section 4.3. Therefore, $W_{\rho}=90, N_{1}=430$ are chosen.

\subsubsection{Forward Transform}

The error results for the forward 2D-DFT of Four-term sinusoid \& Sinc function with $W_{\rho}=90$, $N_{1}=430$ are shown in Figure 17. The discrete transform does not approximate the continuous transform very well. This is expected because the function in the frequency domain is discontinuous and the sampling points close to the discontinuity will result in a very large error. The maximum value of the error is $E r r o r_{\text {max }}=10.6535 \mathrm{~dB}$ and this occurs where the discontinuities are located. The average of the error is Error $r_{\text {average }}=-38.7831 \mathrm{~dB}$.

With $W_{\rho}=90, N_{1}=430$, Table 5 shows the errors with respect to different value of $N_{1}$ and $N_{2}$, from which Figure 18 and Figure 19 show the trend. From Figure 18, increasing $N_{1}$ alone tends improve the average error. The maximum error does not change with $N_{1}$, which is reasonable because of the discontinuity of the function in the frequency domain.

From Figure 19, increasing $N_{2}$ leads to Error $r_{\max }$ and Error ${ }_{\text {average }}$ first improving and then worsening. This is reasonable because when $N_{2}$ is less than the minimum requirement of 31 from sampling theorem, the test result is actually affected by both sampling point density (from the sampling theorem) and grid coverage (discussed in Section 4.3). Increasing $N_{2}$ should give better results from the point of view of the sampling theorem but worse grid coverage. The result from the combined effects is dependent on the function properties. In the specific case of this function, 
588

589

590

when $N_{2}$ is bigger than 31 , thereby implying that the angular sampling theorem has been satisfied - the results get worse with increasing $N_{2}$.

591 The error results for the 2D-IDFT of Four-term sinusoid \& Sinc function with $W_{\rho}=90, N_{1}=430$ 592 are shown in Figure 20. The maximum value of the error is $E r r o r_{\max }=-8.6734 d B$. The average of 593 the error is Error ${ }_{\text {average }}=-37.8119 \mathrm{~dB}$. With $W_{\rho}=90, N_{1}=430$, Table 6 shows the errors with respect to different value of $N_{1}$ and $N_{2}$, from which Figure 21 and Figure 22 show the trend.

From Figure 21, it can be observed that the increasing $N_{1}$ alone improves the average error, as was expected. However, $N_{1}=380$ gives an apparently worse average error than the other points. This could be caused by the discontinuity of the function in the frequency domain. Changing to $N_{1}=381$, the average error becomes $-37.0 \mathrm{~dB}$ which proves that the large error is caused by the discontinuity.

From Figure 22, increasing $N_{2}$ does not lead to worse results, which is different from previous cases. However, from Figure 16 it can be seen that the function in the frequency domain does not have much information in the center area. So, even though increasing $N_{2}$ causes a larger hole in the center as discussed in Section 4.3, it does not lead to losing much energy which explains why Figure 22 shows a different trend from the previous cases.

Performing 2D-DFT and 2D-IDFT sequentially results in $\varepsilon=1.3117 \times e^{-12}$ where $\varepsilon$ is calculated by equation (51) .

6.2.3 Four-term sinusoid and modified exponential

For the next test function, the function is given by

$$
f(r, \theta)=\frac{\mathrm{e}^{-a r}}{r}[3 \sin (\theta)+\sin (3 \theta)+4 \cos (10 \theta)+12 \sin (15 \theta)]
$$

Its continuous 2D-FT can be calculated as

$$
\begin{aligned}
F(\rho, \psi)= & -6 \pi i \sin (\psi) \frac{\sqrt{\rho^{2}+a^{2}}-a}{\rho \sqrt{\rho^{2}+a^{2}}}+2 \pi i \sin (3 \psi) \frac{\left(\sqrt{\rho^{2}+a^{2}}-a\right)^{3}}{\rho^{3} \sqrt{\rho^{2}+a^{2}}} \\
& -8 \pi \cos (10 \psi) \frac{\left(\sqrt{\rho^{2}+a^{2}}-a\right)^{10}}{\rho^{10} \sqrt{\rho^{2}+a^{2}}}+24 \pi i \sin (15 \psi) \frac{\left(\sqrt{\rho^{2}+a^{2}}-a\right)^{15}}{\rho^{15} \sqrt{\rho^{2}+a^{2}}}
\end{aligned}
$$

612 The graphs for the original function and the magnitude of its continuous 2D-FT with $a=0.1$ are 613 shown in Figure 23. From Figure 23, it can be observed that the function has a spike in both 614 domains, which is a more difficult scenario based on the discussion in Section 4.3. In this case, the 615 function can be assumed as space-limited or band-limited. This function will be tested as being 616 space-limited. 
618 effectively space-limited with $R=20$, and $F(\rho, \psi)$ is effectively band-limited with $W_{\rho}=15$,

619 which gives $j_{0 N_{1}} \approx 300$. This results in $N_{1}=96$. However, since the function explodes at the 620 center area in both domains, relatively large values of $R$ and $W_{\rho}$ should give a better 621 approximation. Therefore, another case with $R=40, W_{\rho}=30$ is tested. In this case, $N_{1}=383$ is 622 chosen.

623 In the angular direction, the highest frequency term is $12 \sin (15 \theta)$. From the sampling 624 theorem, the sampling frequency should be at least twice of the highest frequency of signal. Thus, $625 N_{2}=41$ is chosen.

\section{6.2.3.1 Forward Transform}

627 Here, the function is tested as a space limited function and equation (14) and (15) are used to 628 proceed with the forward and inverse transform in sequence. The error results with $629 R=40, W_{\rho}=30, N_{1}=383$ are shown in Figure 24. The maximum value of the error is 630 Error $_{\max }=-10.1535 \mathrm{~dB}$ and this occurs at the center area. The average of the error is 631 Error $_{\text {average }}=-32.7619 \mathrm{~dB}$. This demonstrates that the discrete function approximates the sampled 632 values of the continuous function quite well.

\section{6.2.3.2 Inverse Transform}

634 The error results with $R=40, W_{\rho}=30, N_{1}=383$ are shown in Figure 25.

635 The maximum value of the error is Error $_{\max }=0.5579 \mathrm{~dB}$ and this occurs at the center. The average 636 of the error is Error $_{\text {average }}=-68.7317 \mathrm{~dB}$.

637 Performing 2D-DFT and 2D-IDFT results in $\varepsilon=1.421 \times e^{-12}$, where $\varepsilon$ is calculated by 638 equation (51).

639 It can be observed that even for functions with the worst properties, the proposed transform 640 can still be used to approximate the continuous Fourier transform with fairly small errors, as long 641 as the function is sampled properly.

\section{Summary and Conclusion}

6437.1 Accuracy and Precision of the transform

644 The proposed discrete 2D-Fourier Transform in polar coordinates demonstrates an acceptable 645 accuracy in providing discrete estimates to the continuous Fourier transform in polar coordinates. 646 In [21],[24]and[28], the one dimensional Hankel transform of a sinc function showed similar 647 dynamic error, which could be used as a comparative measure. Since the discrete Hankel transform 648 is one step of the proposed discrete 2D-Fourier Transform, and the definition of the Hankel 649 transform is based on [8], a similar dynamic error should be expected. 
650

651

652

653

654

655

656

657

658

659

660

661

662

663

664

665

666

667

668

669

670

671

672

673

674

675

676

677

678

679

680

681

682

683

684

685

686

687

688

689

690

691
The test cases showed that the transform introduced very small errors $\left(\varepsilon=1.4004 \times e^{-12}\right.$ for worst case) by performing a forward transform and an inverse transform sequentially, which demonstrates that the discrete transform shows good precision.

\subsection{Guidelines of choosing sample size}

As discussed in Section 4.3 and proved by test cases, the sample size $N_{1}$ (sample size in the radial direction) and $N_{2}$ (sample size in the angular direction) do not have to be of the same order. For functions with different properties, sample size in the different directions could be very different. To approximate the continuous Fourier transform properly, sample size should be chosen based on the discussion in Section 4.3.

\subsection{Interpretation of the transform}

By interpreting the transform as a 1DDFT, 1D Discrete Hankel transform and 1D inverse DFT, the computing time of the transform is improved to a useful level since the FFT can be used to compute the DFT.

\section{References}

[1] J. W. Cooley and J. W. Tukey, "An Algorithm for the Machine Calculation of Complex Fourier Series," Math. Comput., vol. 19, no. 90, pp. 297-301, 1965.

[2] Y. Xu, D. Feng, and L. V. Wang, "Exact frequency-domain reconstruction for thermoacoustic tomography. I. Planar geometry," Med. Imaging IEEE Trans. On, vol. 21, no. 7, pp. 823-828, 2002.

[3] M. C. Scott, C.C. Chen, M. Mecklenburg, C. Zhu, R. Xu, P. Ercius, U. Dahmen, B. C. Regan and J. Miao, "Electron tomography at 2.4-ångström resolution," Nature, vol. 483, no. 7390, p. 444, Mar. 2012.

[4] B.P. Fahimian, Y. Zhao, Z. Huang, R. Fung, Y. Mao, C. Zhu, M. Khatonabadi, J.J. DeMarco, S.J. Osher, M.F. McNitt-Gray, J. Miao, "Radiation dose reduction in medical x-ray CT via Fourier-based iterative reconstruction," Med. Phys., vol. 40, no. 3, p. n/a-n/a, Mar. 2013.

[5] E. Lee, B.P. Fahimian, C.V. Iancu, C. Suloway, G.E. Murphy, E.R. Wright, D. Castaño-Díez, G. J. Jensen, J. Miao," Radiation dose reduction and image enhancement in biological imaging through equally-sloped tomography ", J. Struct. Biol., vol. 164, no. 2, pp. 221-227, 2008.

[6] R. M. Lewitt and S. Matej, "Overview of methods for image reconstruction from projections in emission computed tomography," Proc. IEEE, vol. 91, no. 10, pp. 1588-1611, Oct. 2003.

[7] A. Averbuch, R. R. Coifman, D. L. Donoho, M. Elad, and M. Israeli, "Fast and accurate Polar Fourier transform," Appl. Comput. Harmon. Anal., vol. 21, no. 2, pp. 145-167, 2006.

[8] S. A. Abbas, Q. Sun, and H. Foroosh, "An Exact and Fast Computation of Discrete Fourier Transform for Polar and Spherical Grid," IEEE Trans. Signal Process., vol. 65, no. 8, pp. 2033-2048, Apr. 2017.

[9] M. Fenn, S. Kunis, and D. Potts, "On the computation of the polar FFT," Appl. Comput. Harmon. Anal., vol. 22, no. 2, pp. 257-263, Mar. 2007.

[10] A. Dutt and V. Rokhlin, "Fast Fourier Transforms for Nonequispaced Data," SIAM J. Sci. Comput., vol. 14, no. 6, pp. 1368-1393, Nov. 1993.

[11] K. Fourmont, "Non-Equispaced Fast Fourier Transforms with Applications to Tomography," J. Fourier Anal. Appl., vol. 9, no. 5, pp. 431-450, 2003. 
692 [12] A. Dutt and V. Rokhlin, "Fast Fourier Transforms for Nonequispaced Data, II," Appl.

693

694

695

696

697

698

699

700

701

702

703

704

705

706

707

708

709

710

711

712

713

714

715

716

717

718

719

720

721

722

723

724

725

726

727

728

729

730

731

732

733 Comput. Harmon. Anal., vol. 2, no. 1, pp. 85-100, Jan. 1995.

[13] D. Potts, G. Steidl, and M. Tasche, "Fast Fourier Transforms for Nonequispaced Data: A Tutorial," in Modern Sampling Theory: Mathematics and Applications, J. J. Benedetto and P. J. S. G. Ferreira, Eds. Boston, MA: Birkhäuser Boston, 2001, pp. 247-270.

[14] J. A. Fessler and B. P. Sutton, "Nonuniform fast Fourier transforms using min-max interpolation," IEEE Trans. Signal Process., vol. 51, no. 2, pp. 560-574, 2003.

[15] G. Plonka, D. Potts, G. Steidl, and M. Tasche, Numerical Fourier Analysis. Birkhäuser Basel, 2018.

[16] H. Stark, "Sampling theorems in polar coordinates," JOSA, vol. 69, no. 11, pp. 1519-1525, Nov. 1979.

[17] H. Stark and M. Wengrovitz, "Comments and corrections on the use of polar sampling theorems in CT," IEEE Trans. Acoust. Speech Signal Process., vol. 31, no. 5, pp. 1329-1331, Oct. 1983.

[18] N. Baddour, "Discrete Two Dimensional Fourier Transform in Polar Coordinates Part I: Theory and Operational Rules," Mathematics, vol. 7, no. 8, p. 698, Aug. 2019.

[19] R. Bracewell, The Fourier Transform and its Applications. New York: McGraw-Hill, 1999.

[20] C. E. Shannon, "Communication in the presence of noise," Proc. IEEE, vol. 72, no. 9, pp. 1192-1201, 1984.

[21] N. Baddour and U. Chouinard, "Theory and operational rules for the discrete Hankel transform," JOSA A, vol. 32, no. 4, pp. 611-622, Apr. 2015.

[22] D. W. Lozier, "NIST Digital Library of Mathematical Functions," Ann. Math. Artif. Intell., vol. 38, no. 1-3, pp. 105-119, 2003.

[23] N. Baddour, "The Discrete Hankel Transform," in Fourier Transforms - Century of Digitalization and Increasing Expectations, London, UK: IntechOpen, 2019.

[24] M. Guizar-Sicairos and J. C. Gutiérrez-Vega, "Computation of quasi-discrete Hankel transforms of integer order for propagating optical wave fields," JOSA A, vol. 21, no. 1, pp. 53-58, Jan. 2004.

[25] N. Baddour and U. Chouinard, "Matlab Code for the Discrete Hankel Transform," J. Open Res. Softw., vol. 5, no. 1, Jan. 2017.

[26] A. D. Poularikas, Transforms and Applications Handbook, Third Edition. CRC Press, 2010.

[27] N. Baddour, "Two-Dimensional Fourier Transforms in Polar Coordinates," Adv. Imaging Electron Phys., vol. 165, pp. 1-45, Jan. 2011.

[28] W. Higgins and Jr. Munson D., "An algorithm for computing general integer-order Hankel transforms," Acoust. Speech Signal Process. IEEE Trans. On, vol. 35, no. 1, pp. 86-97, 1987.

\section{Appendix: Improving the computing time of the transform}

One of the advantages of the traditional Fourier transform is that the computing speed is fast by using the now well-established $f f t$ algorithm. To reduce the computing time of the 2D DFT in polar coordinates, the following steps are recommended: 
734

735

736

737

738

739

740

741

742

743

744

745

746

747

748

749

750

751

752

753

754

755

756

757 758

759 760

1. Interpreting the transform as three sequential operations (DFT, DHT, IDFT) instead of a single four-dimensional matrix.

2. Pre-calculating and saving the Bessel zeros.

8.1 Reducing computing time by interpreting the transform as three operations in sequence

As explained above, the essence of the transform is that the matrix $f_{p k}$ is transformed into the matrix $F_{q l}$. The intuitive way to interpret the transform kernel is to think of it as a four-dimensional matrix or matrices in a matrix. However, interpreting the transform as a 1D-DFT of each column, a 1D-DHT of each row and then a 1D-IDFT of each column makes it possible to use the Matlab built in functions $f f t$ and ifft, which significantly reduced the computational time.

\subsection{Reduce computing time by pre-calculating the Bessel Zeros}

After defining the transform as three operations in sequence and using the matrix for the discrete Hankel transform defined in [21], it was found that a lot of computational time was used to calculate the Bessel zeros for every different test case, even though the Bessel zeros are the same in every case. Pre-calculating the Bessel zeros and storing the results for large numbers of $N_{1}$ and $N_{2}$ saves a lot of time.

Table 7 shows the computing time of a forward transform on the same computer (Processor: Intel(R) Core(TM) i7-4710HQ CPU, RAM:12GB) for three cases:

1. Evaluate the transform as matrices in a matrix without pre-calculating the Bessel zeros.

2. Evaluate the transform as a DFT, DHT and IDFT in sequence without pre-calculating the Bessel zeros.

3. Evaluate the transform as a DFT, DHT and IDFT in sequence with pre-calculating the Bessel zeros.

The Gaussian function was used as the test function therefore $N_{1}=383$ and $N_{2}=15$.

From Table 7, it can be clearly observed that the computing time by running the transform as matrices in a matrix costs $3346.5 \mathrm{~s}$, which is not acceptable or the transform to be useful. Testing 
761 the transform as three operations turns 3346.5 seconds into 321.1 seconds. This is much better.

762 Finally, pre-calculating the Bessel Zeros makes the transform much faster and applicable.

763 
Figure 1

Spatial sampling grid for a space-limited function with $R=1, N_{1}=16$ and $N_{2}=15$

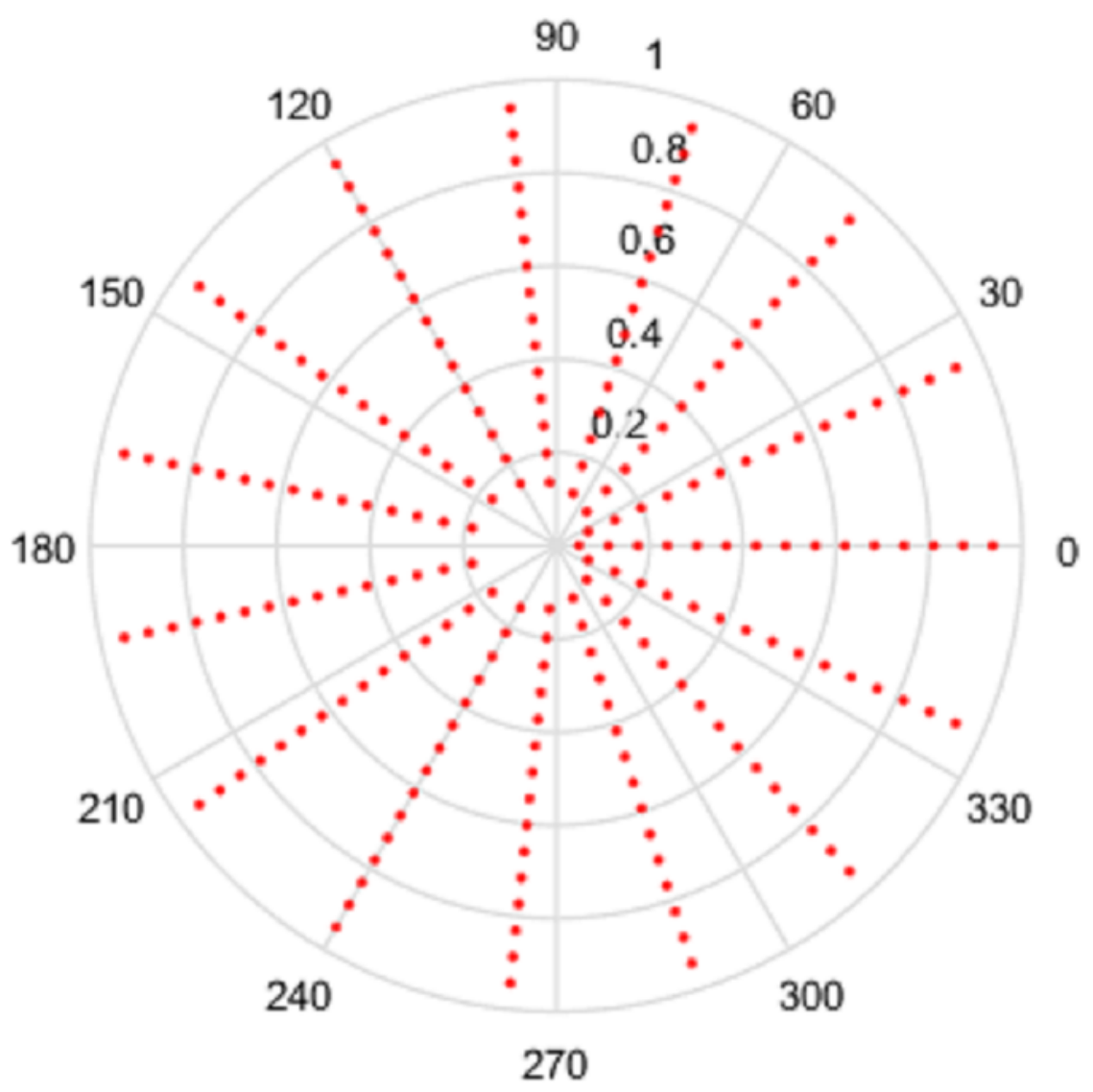


Figure 2

Frequency space sampling grid for a space-limited function with $R=1, N_{1}=16$ and $N_{2}=15$

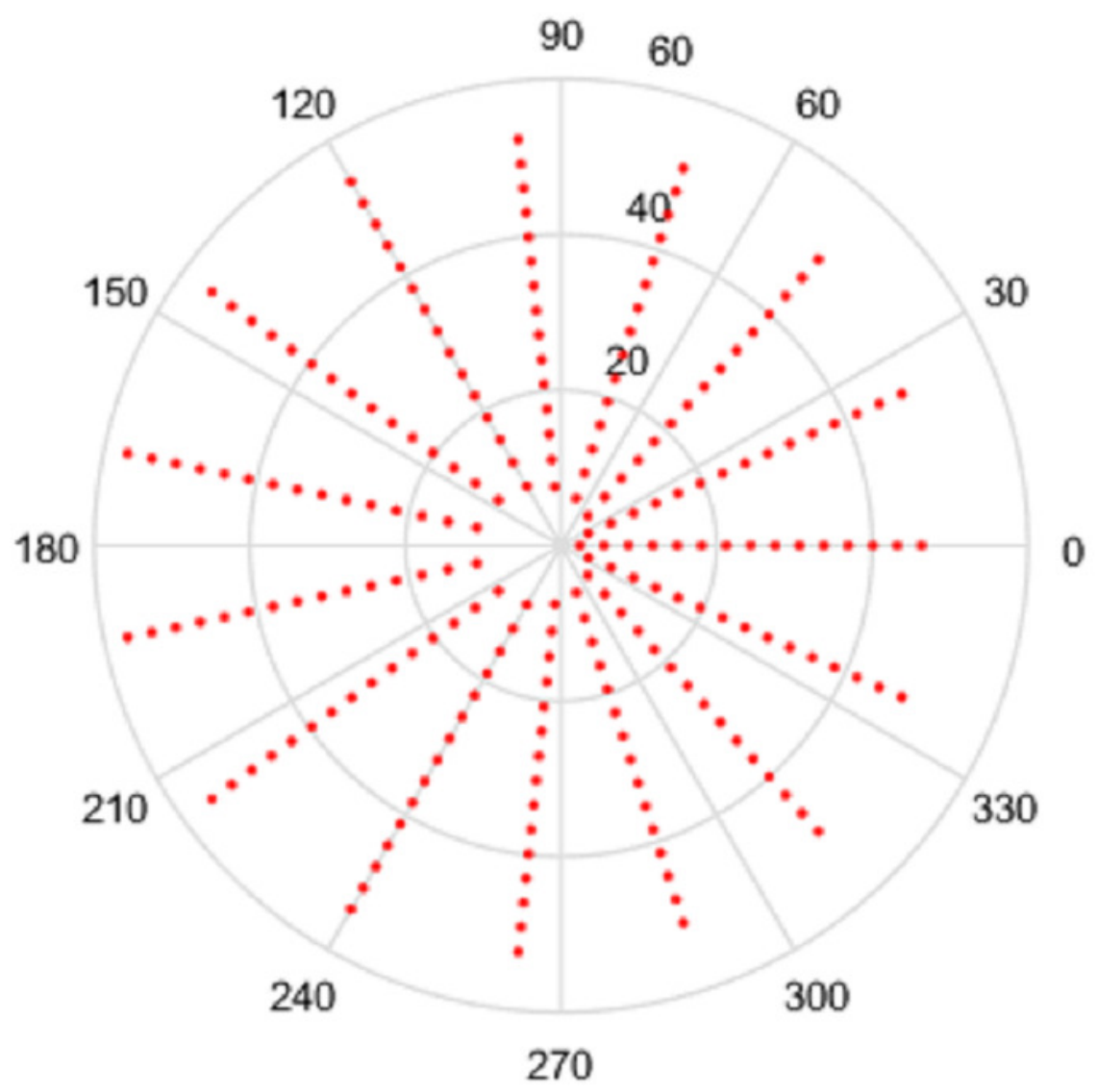


Figure 3

Spatial sampling grid for a space-limited function with $R=1, N_{1}=96$ and $N_{2}=95$

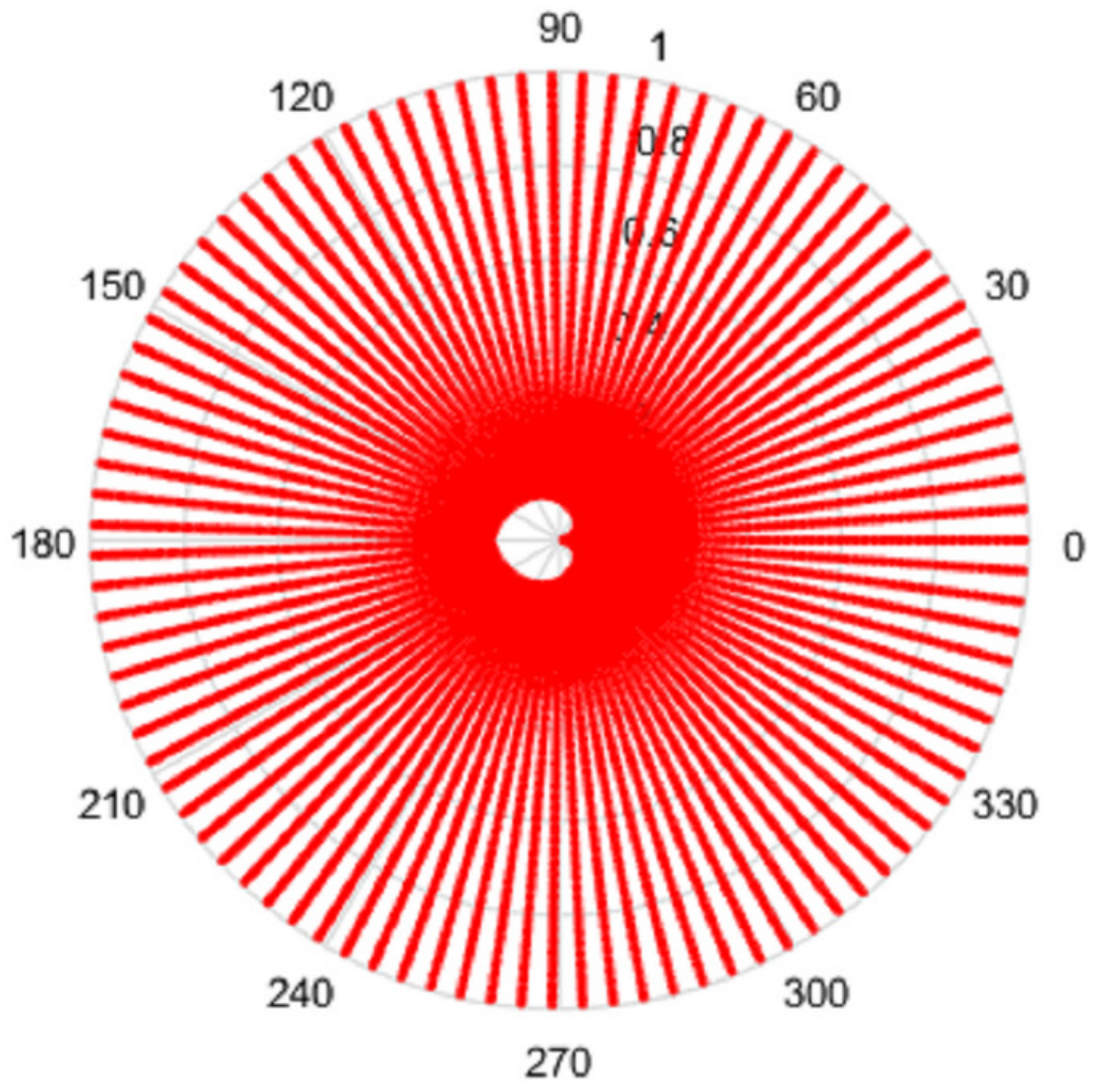


Figure 4

Frequency space sampling grid for a space-limited function with $R=1, N_{1}=96$ and $N_{2}=95$

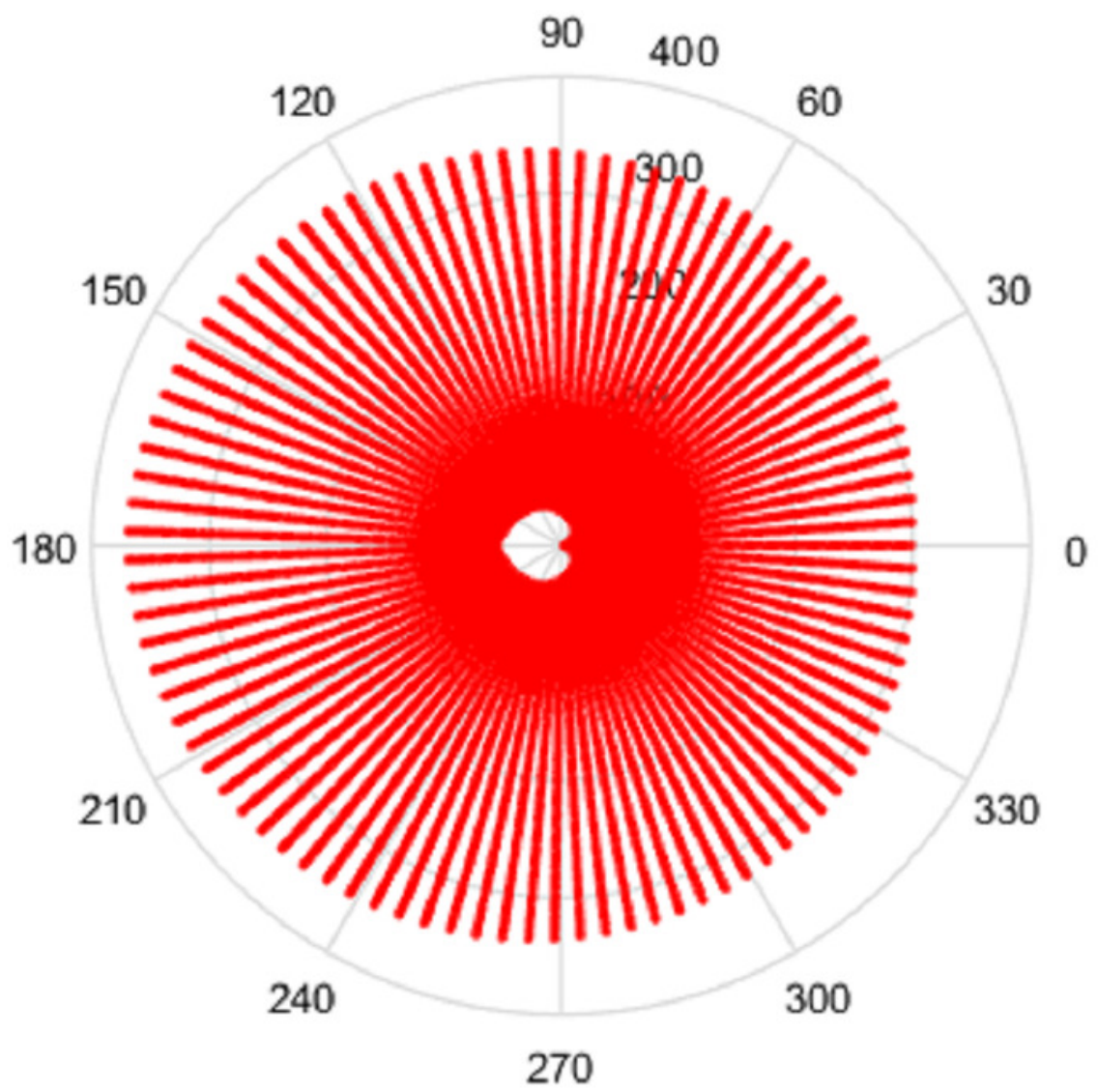




\section{Figure 5}

(a) Original function (Gaussian) and (b) its continuous 2D-DFT (which is also a Gaussian)

(a) Original Function

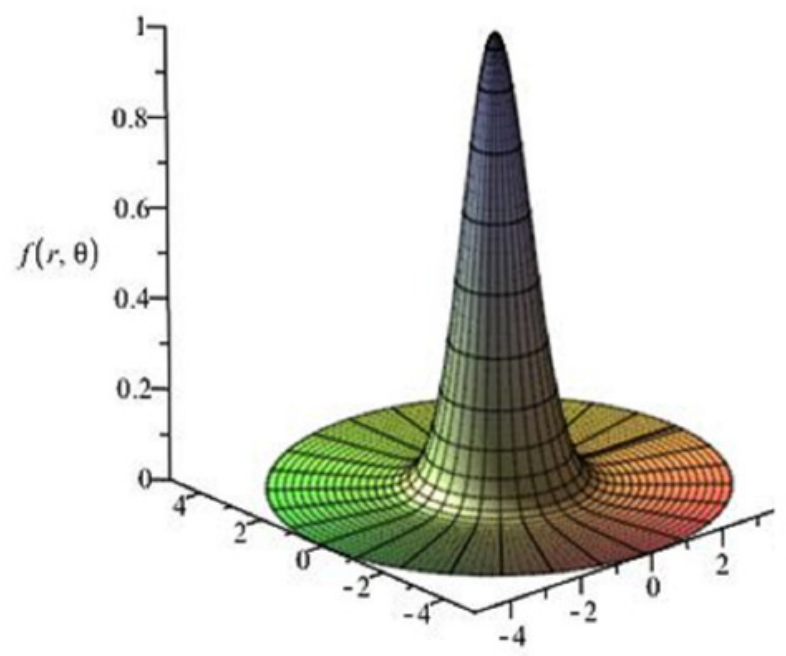

(b) 2D-Fourier Transform

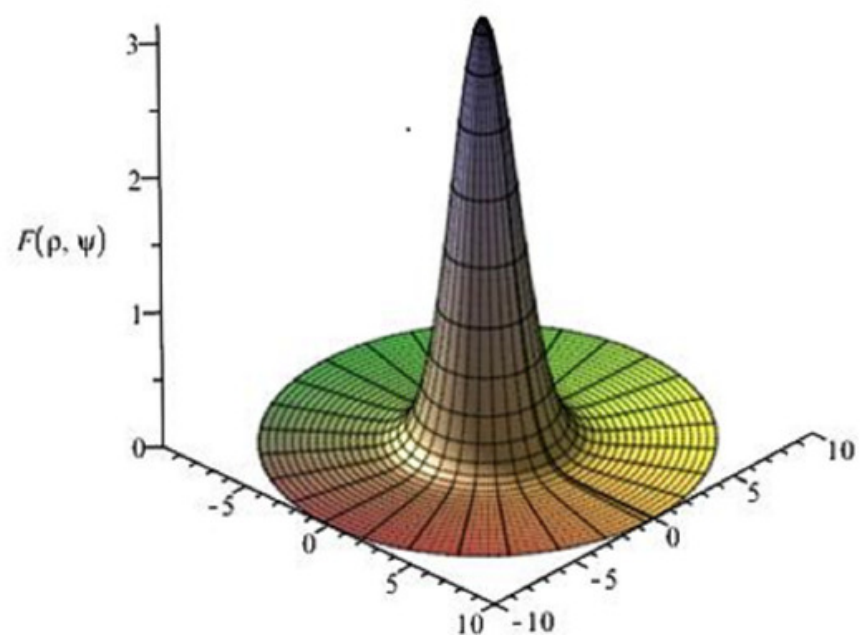




\section{Figure 6}

(a) sampled continuous transform and (b) discrete forward transform for a Gaussian function with $\mathrm{R}=5$ and $\mathrm{N}_{1}=17$

(a) Sampled Continuous Forward Transform

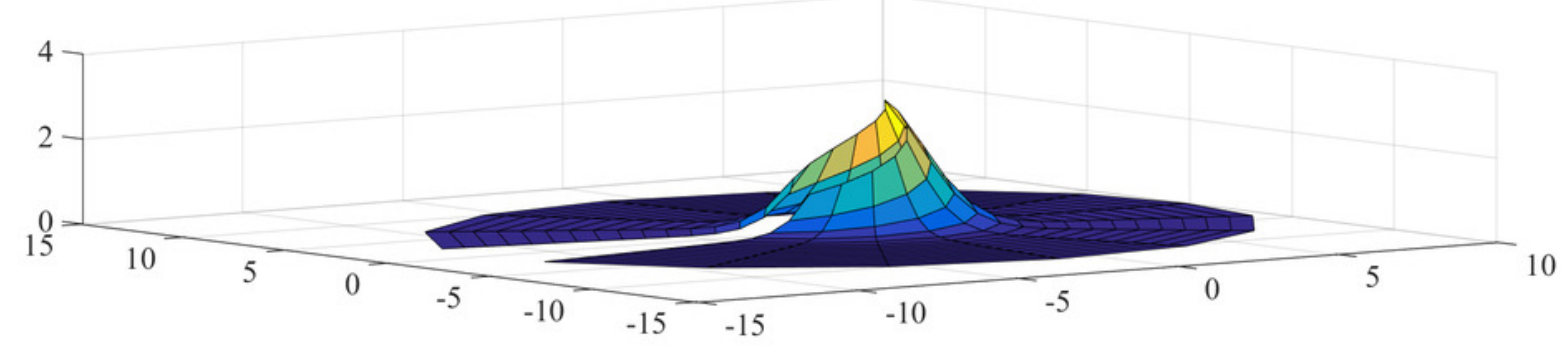

(b) Discrete Forward Transform

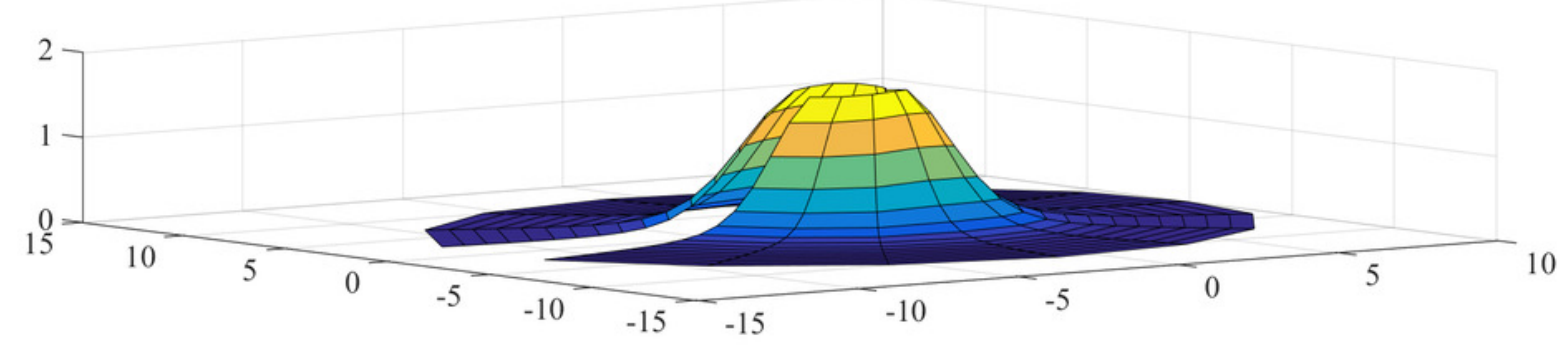




\section{Figure 7}

Error between the sampled values of the continuous transform and the discretely calculated values for a Gaussian function with $\mathrm{R}=5$ and $\mathrm{N}_{1}=17$

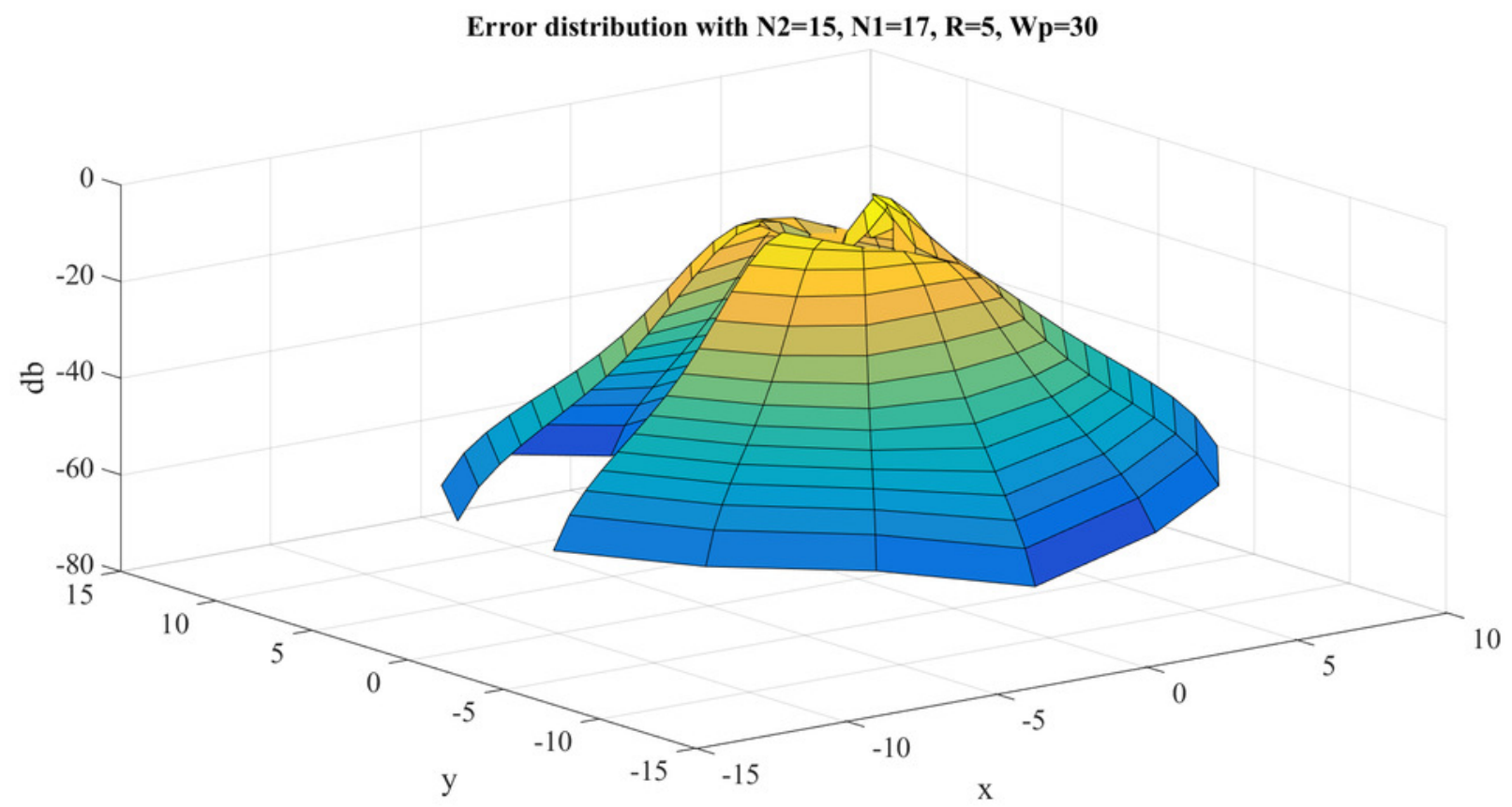




\section{Figure 8}

Error between the sampled values of the continuous transform and the discretely calculated values for a Gaussian function with $R=40$ and $N_{1}=383$

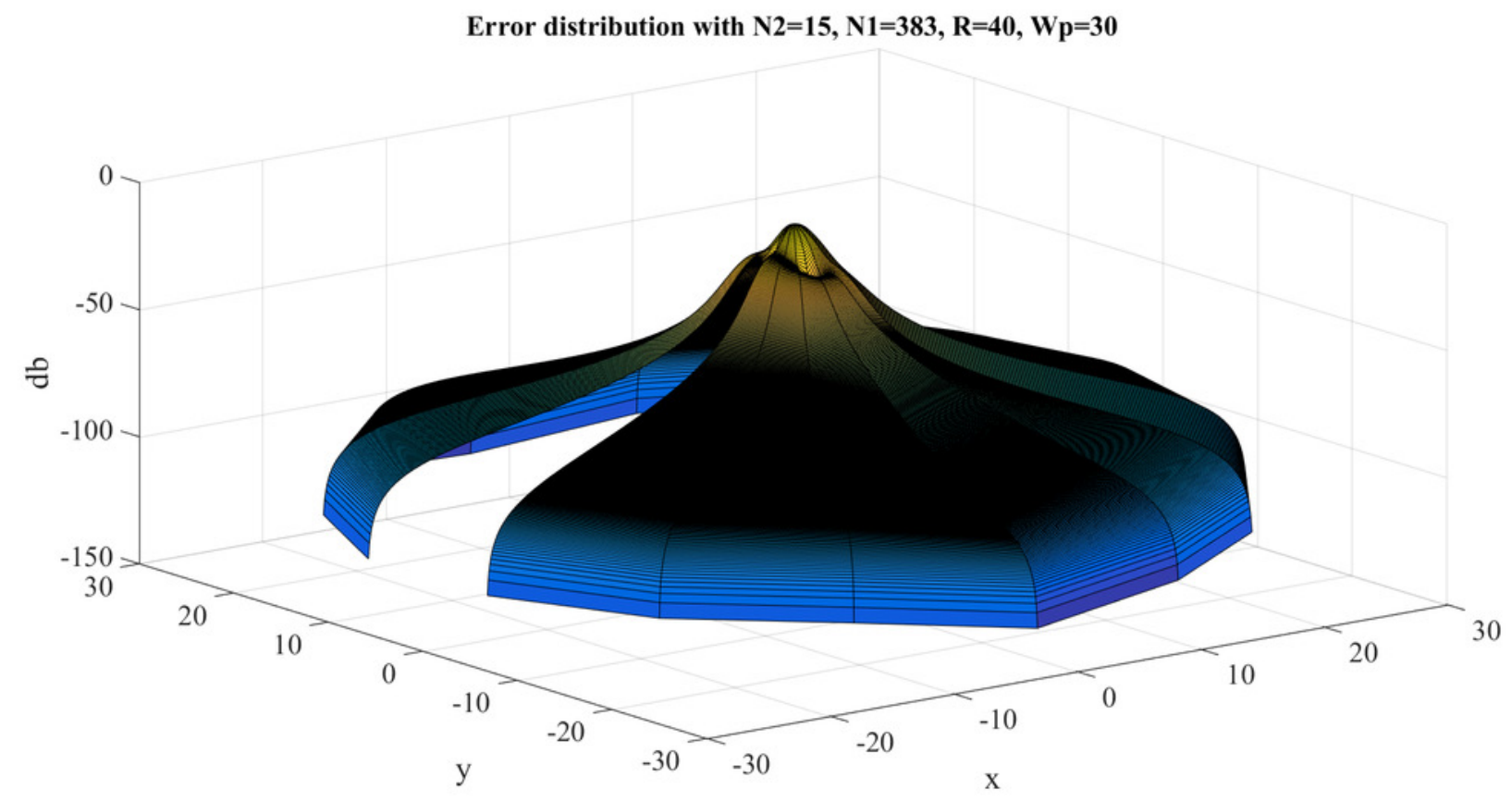


Figure 9

Error trend between the sampled values of the continuous transform and the discretely calculated values for a Gaussian function, as a function of $\mathrm{N}_{1}$

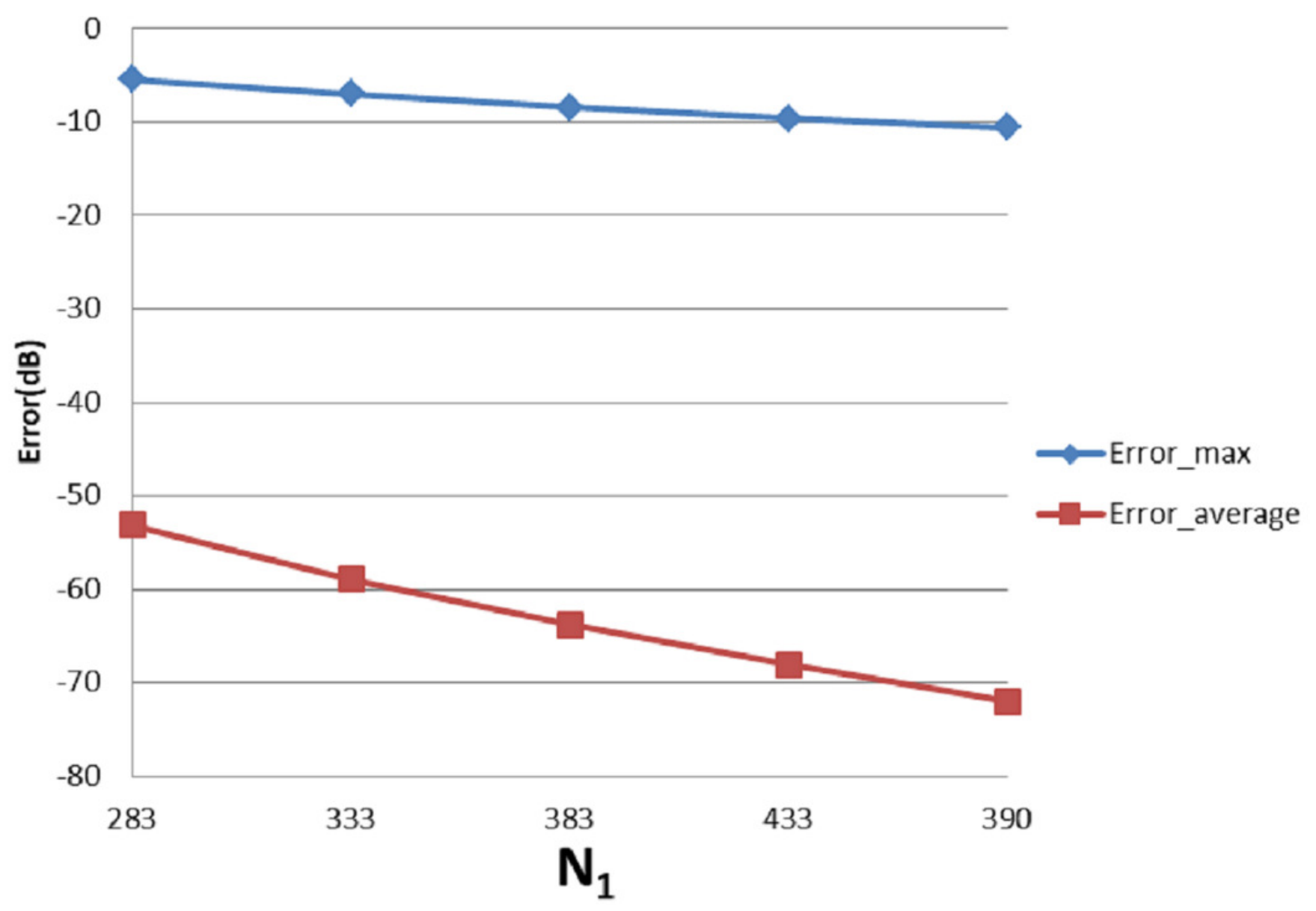


Figure 10

Error trend between the sampled values of the continuous transform and the discretely calculated values for a Gaussian function, as a function of $\mathrm{N}_{2}$

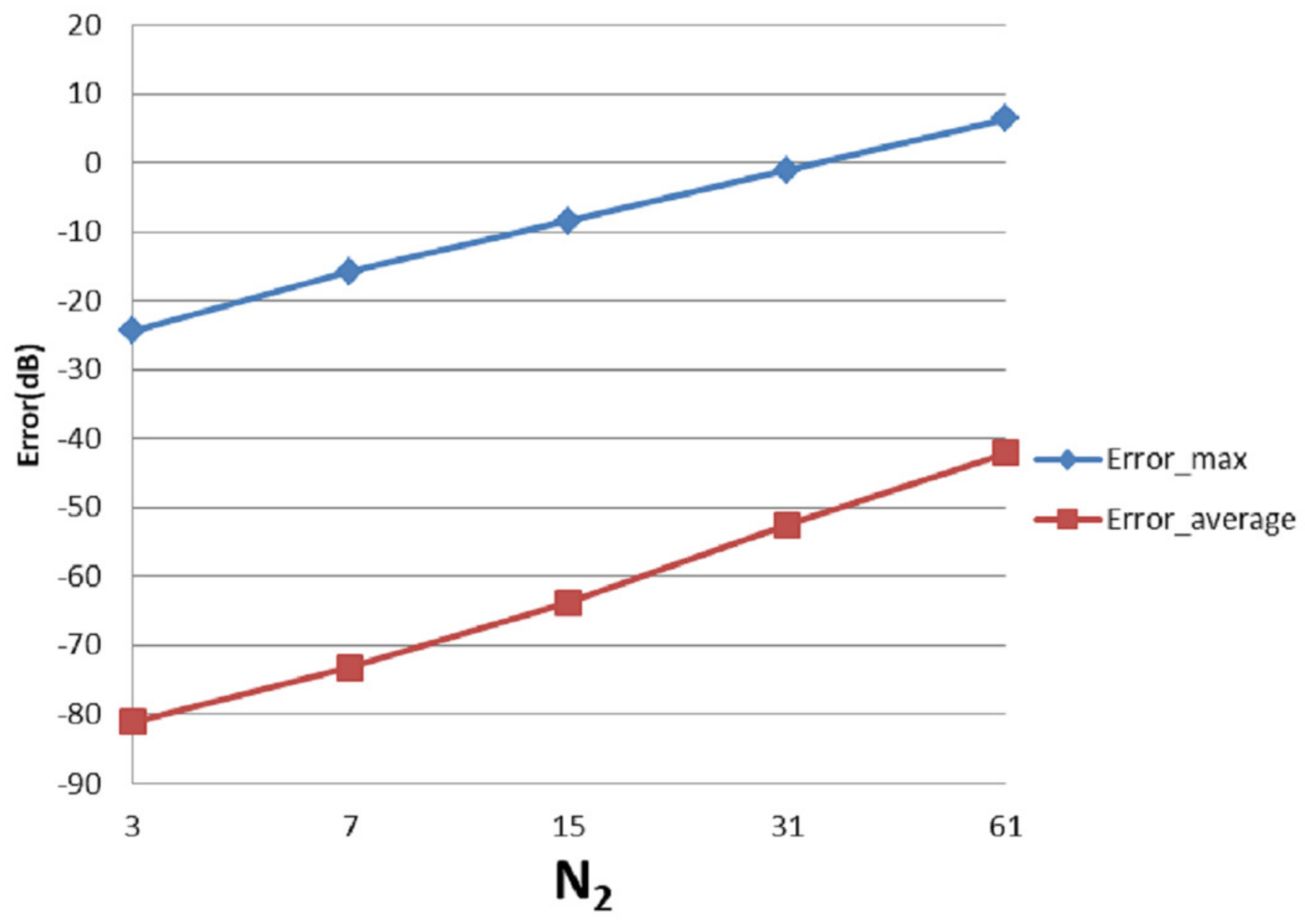




\section{Figure 11}

(a) sampled continuous inverse transform and (b) discrete inverse transform for the Gaussian function for $\mathrm{R}=5$ and $\mathrm{N}_{1}=17$

(a) Continuous Inverse Transform

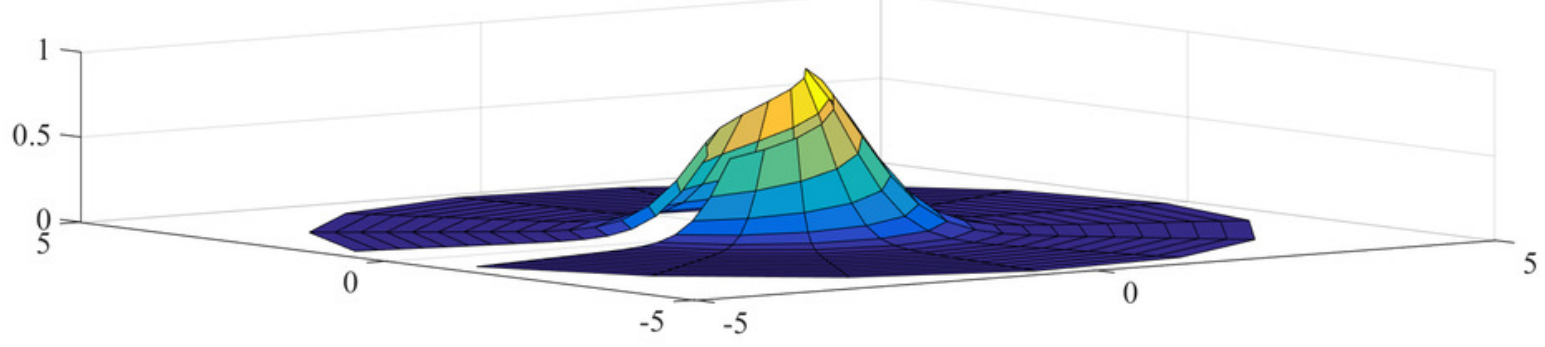

(b) Discrete inverse Transform

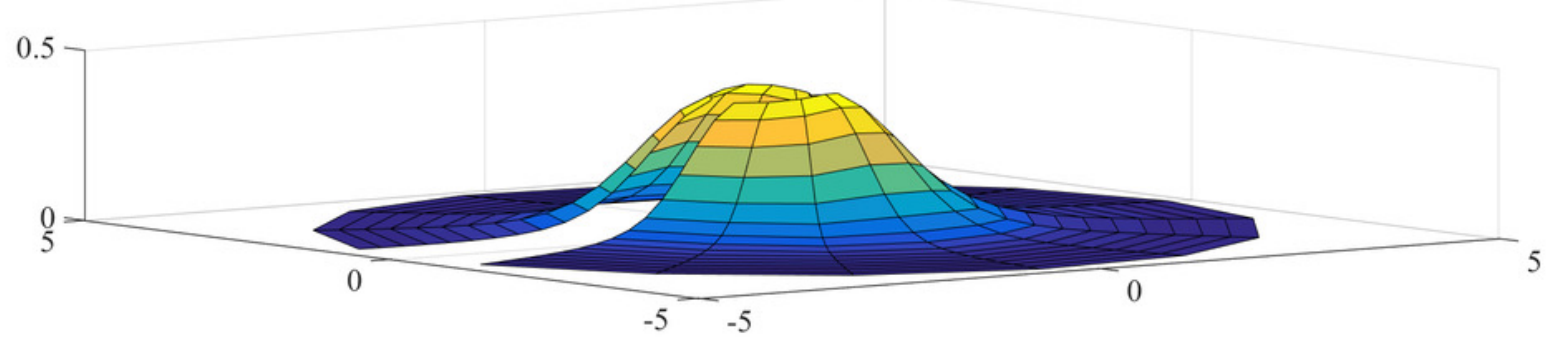




\section{Figure 12}

Error between the sampled continuous inverse transform and discrete inverse transform for the Gaussian function for $\mathrm{R}=5$ and $\mathrm{N}_{1}=17$

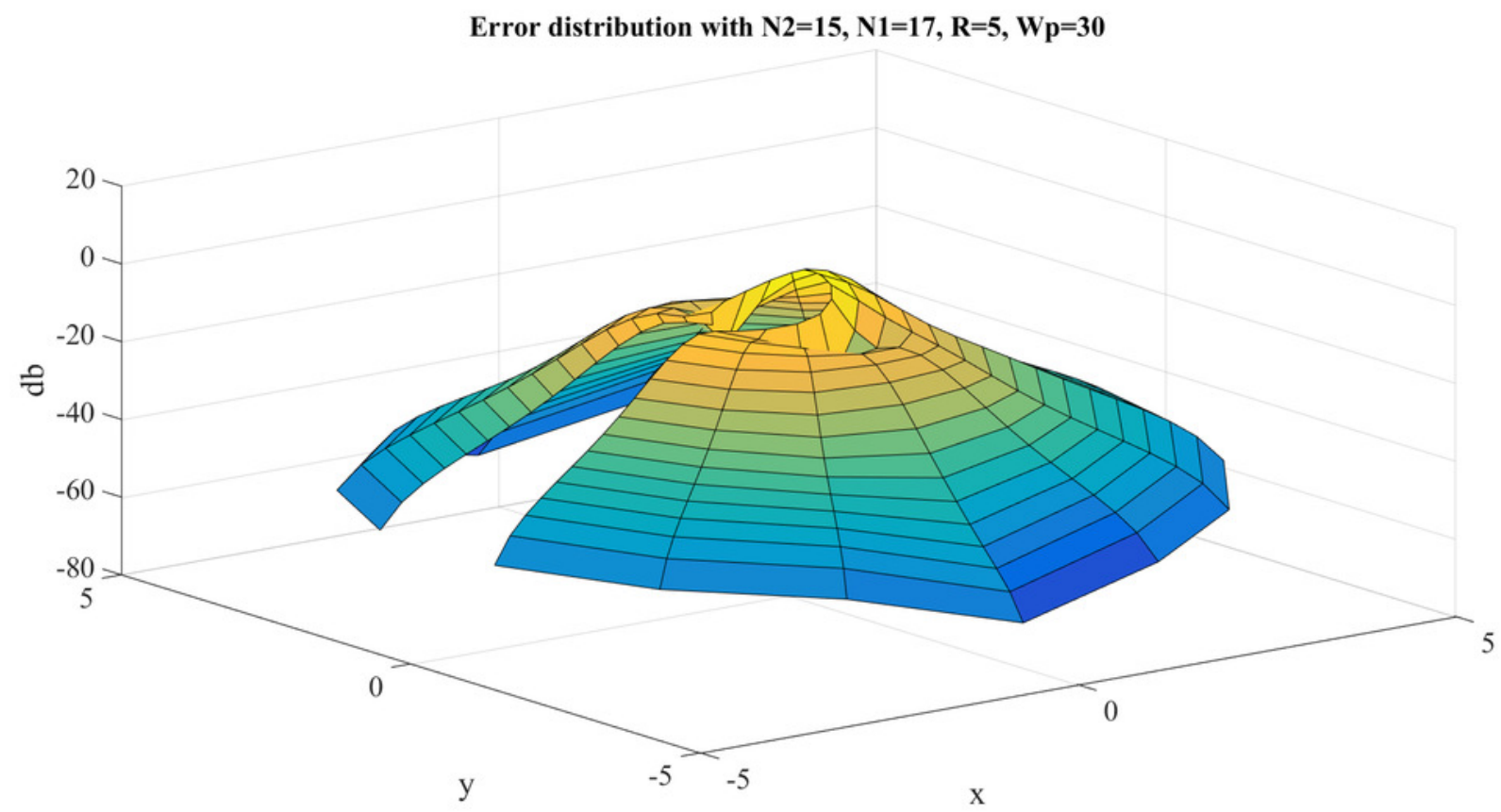




\section{Figure 13}

Error between the sampled continuous inverse transform and discrete inverse transform for the Gaussian function for $\mathrm{R}=40$ and $\mathrm{N}_{1}=383$

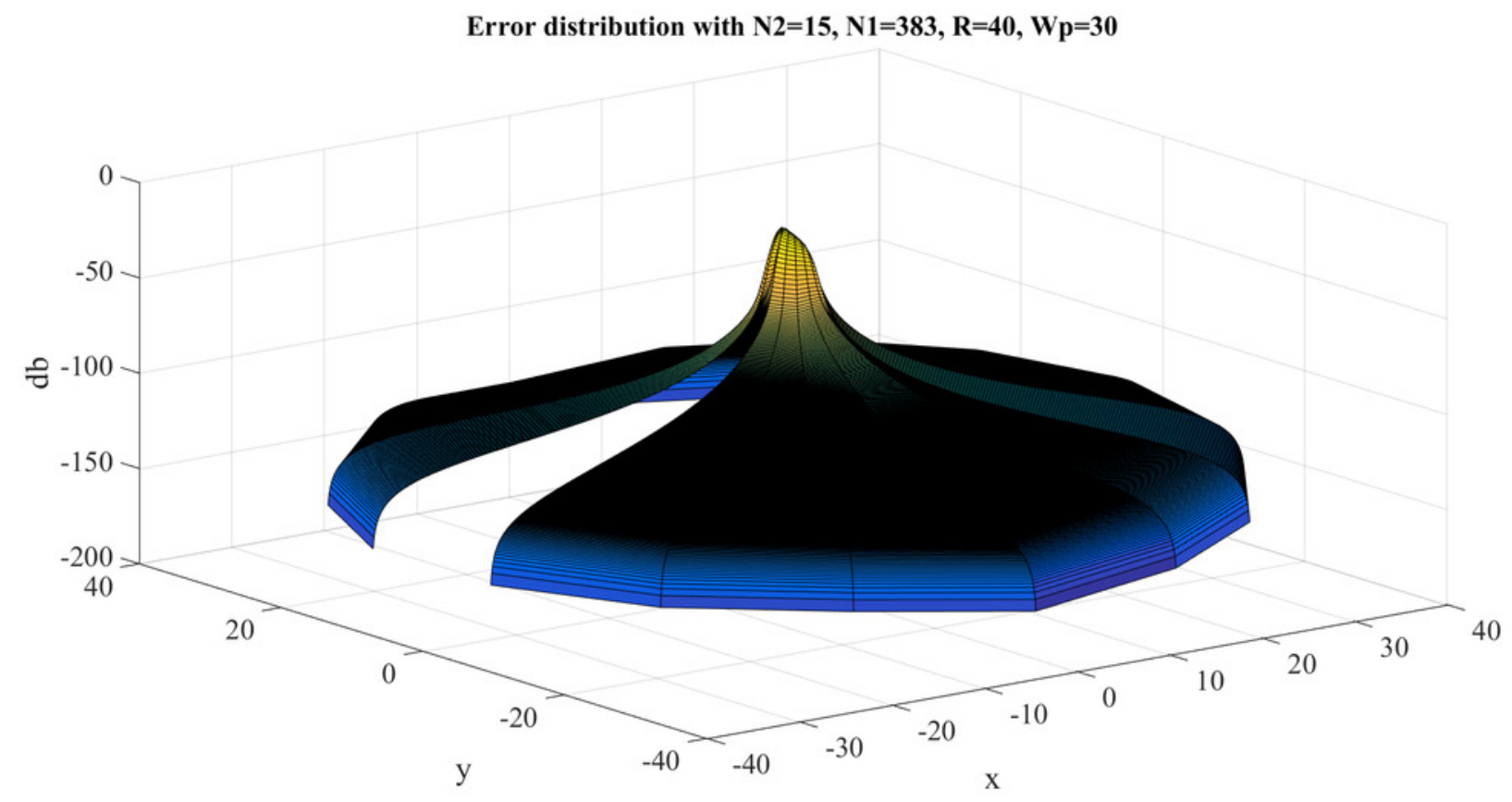


Figure 14

Error trend between the sampled values of the continuous inverse transform and the discretely calculated values for a Gaussian function, as a function of $N_{1}$

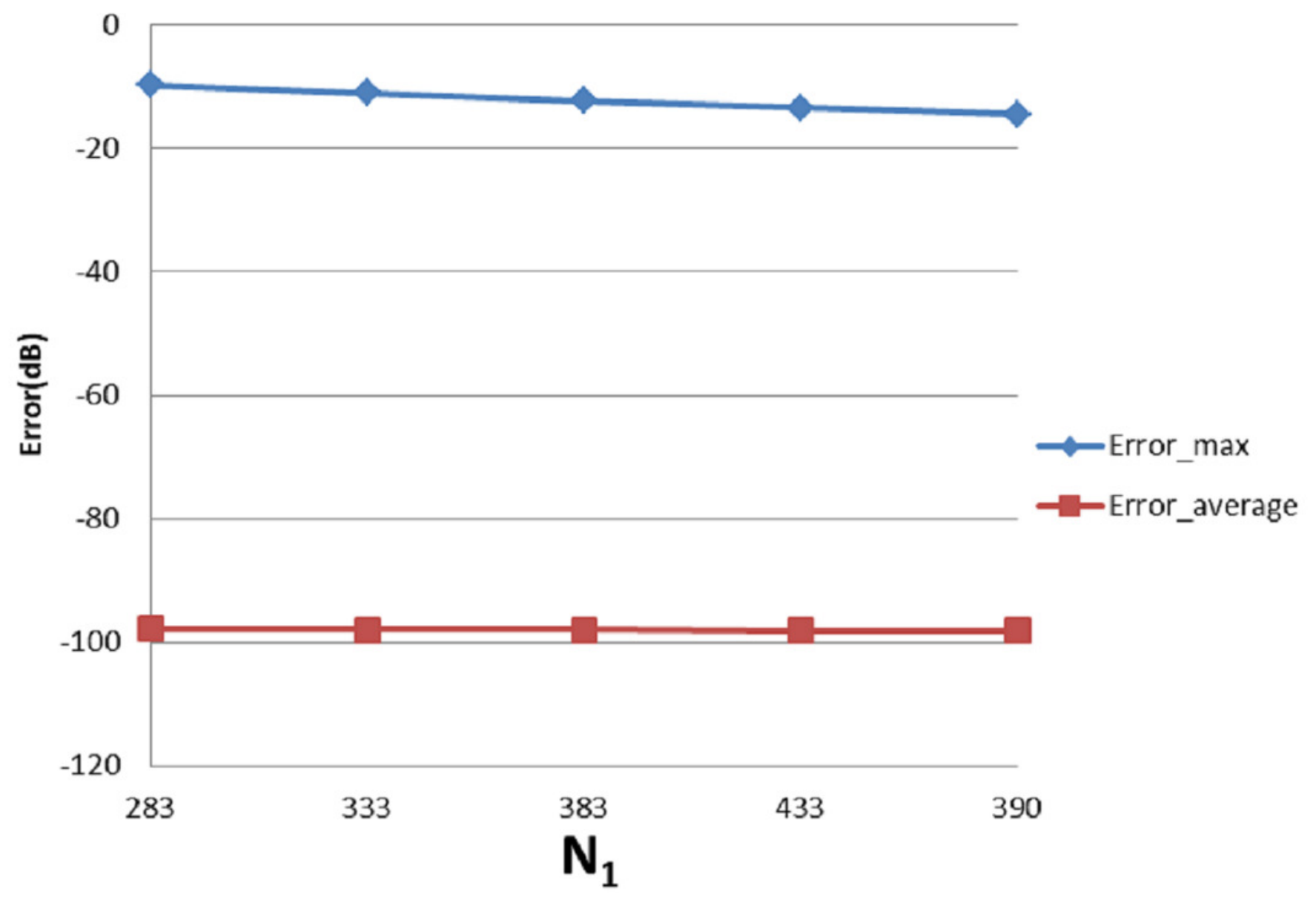


Figure 15

Error trend between the sampled values of the continuous inverse transform and the discretely calculated values for a Gaussian function, as a function of $\mathrm{N}_{2}$

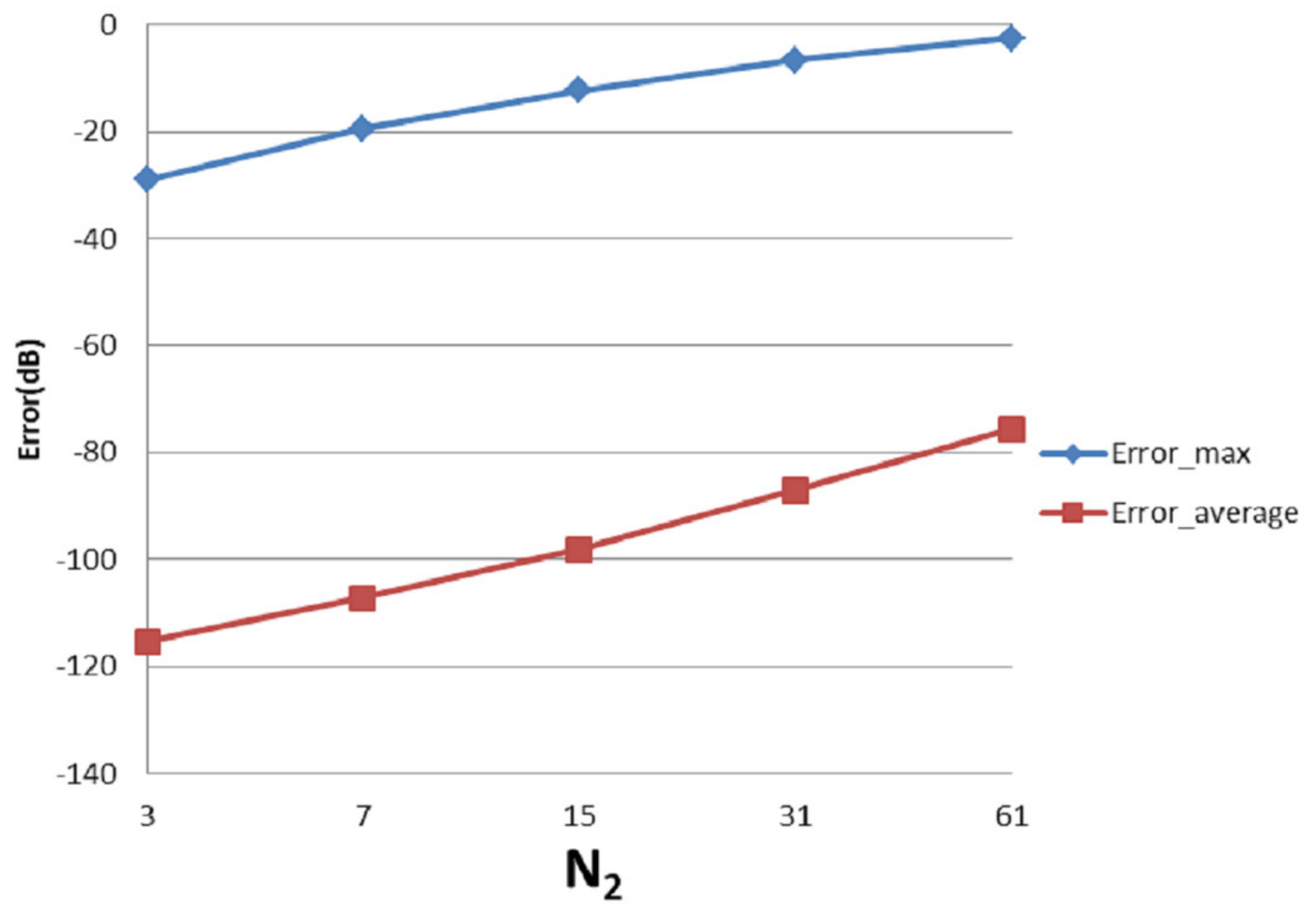


Figure 16

Plots of the (a) original function (four-term sinusoid and sinc) and (b) the magnitude of its continuous forward 2D Fourier transform with $a=5$

(a) Original Function

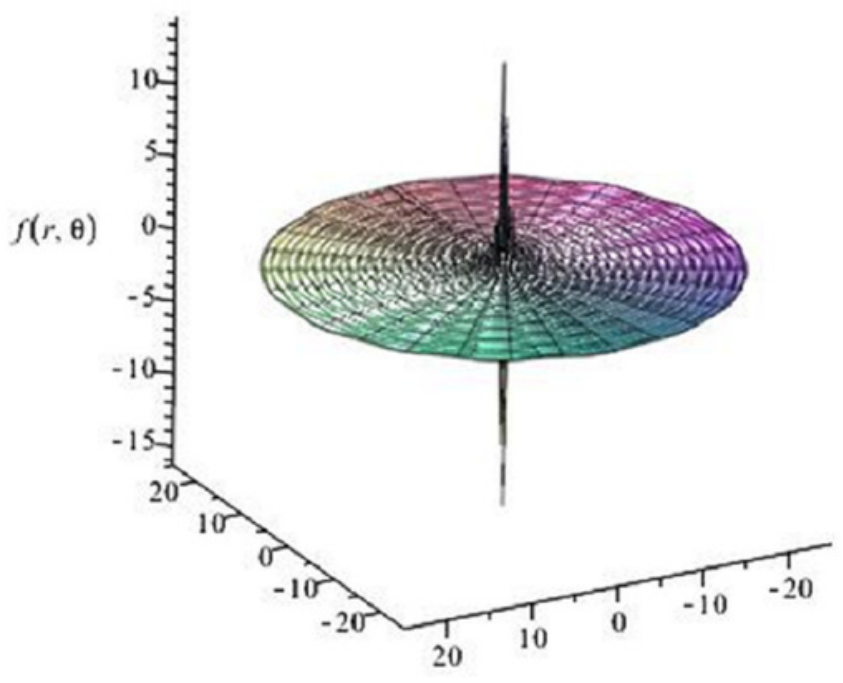

(b) 2D-Fourier Transform

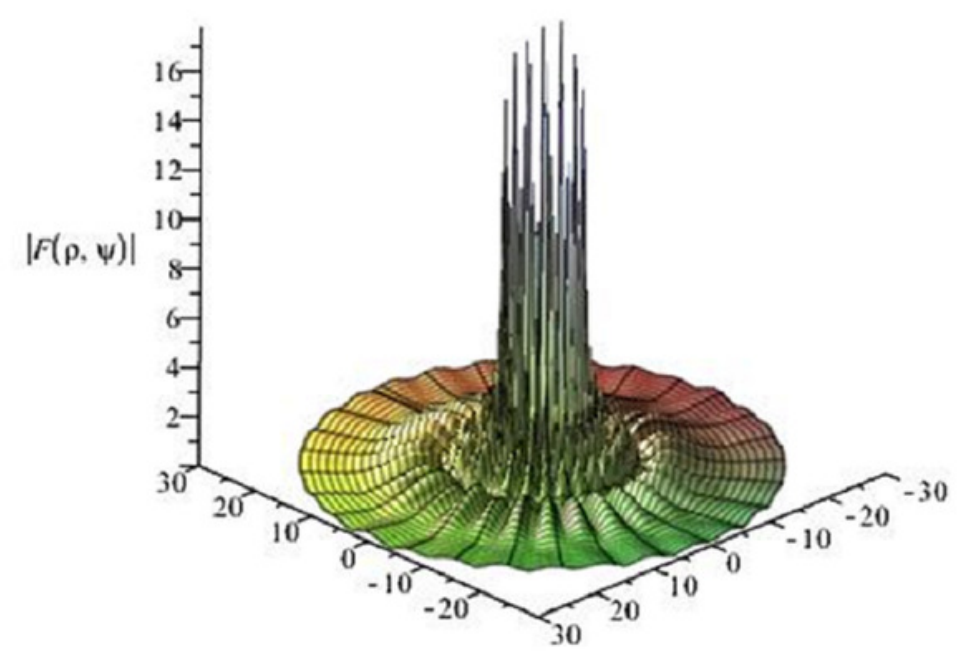




\section{Figure 17}

Error results for the forward 2D Fourier transform of the Four-term sinusoid \& Sinc function for $\mathrm{W}_{p}=90$ and $\mathrm{N}_{1}=430$

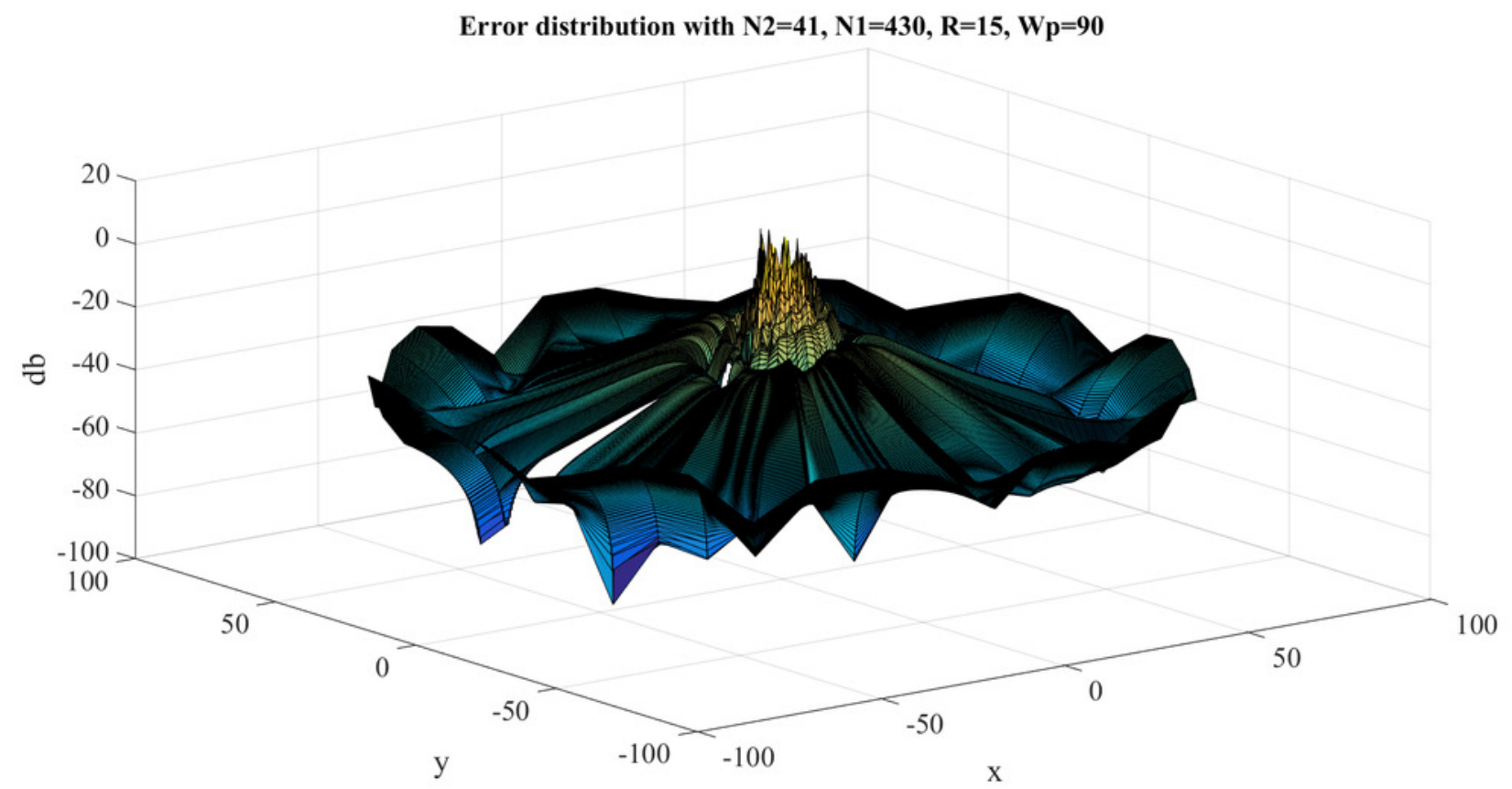


Figure 18

Error trend between the sampled values of the continuous forward transform and the discretely calculated values for a Four-term sinusoid and sinc as a function of $\mathrm{N}_{1}$

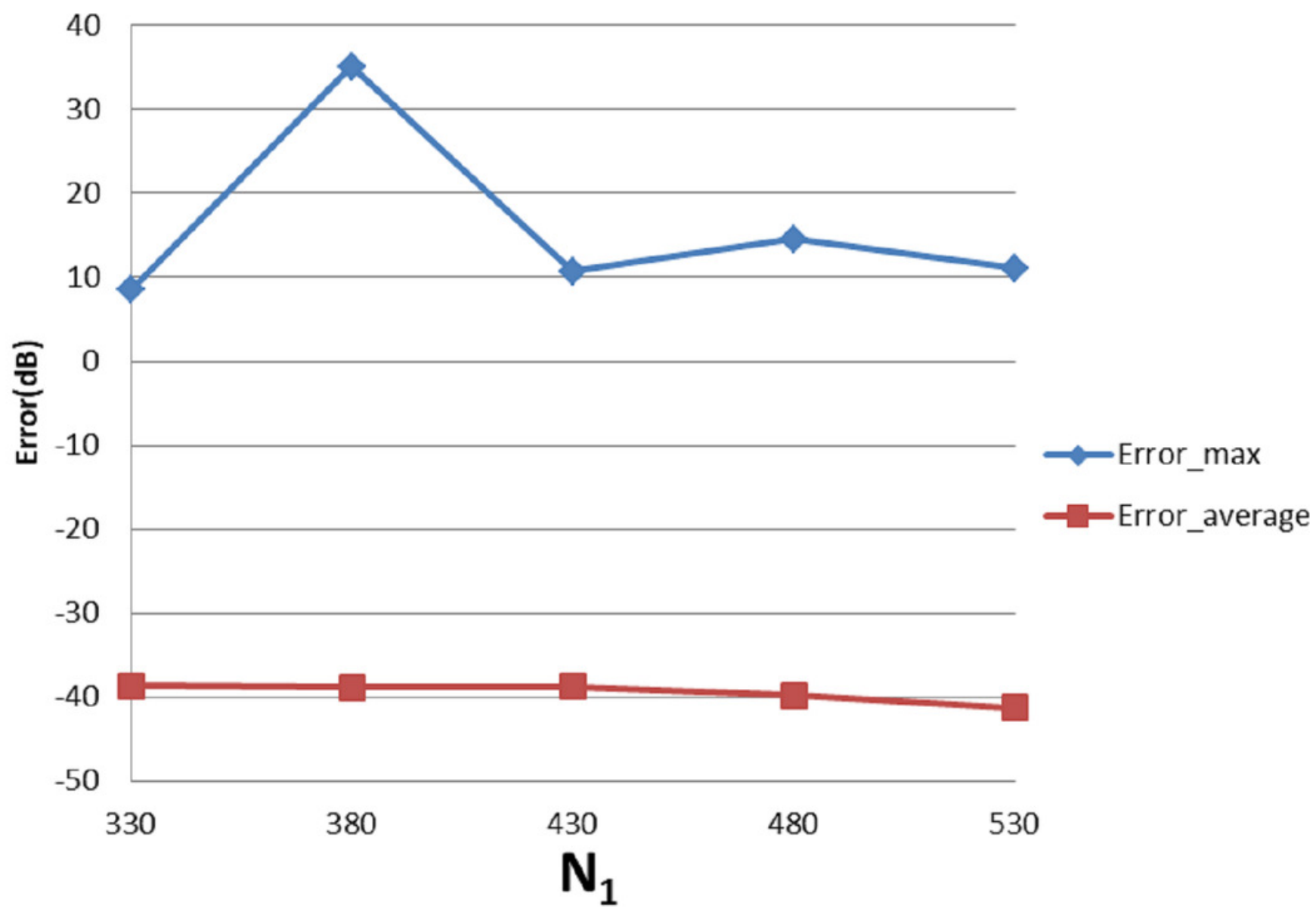


Figure 19

Error trend between the sampled values of the continuous forward transform and the discretely calculated values for a Four-term sinusoid and sinc as a function of $\mathrm{N}_{2}$

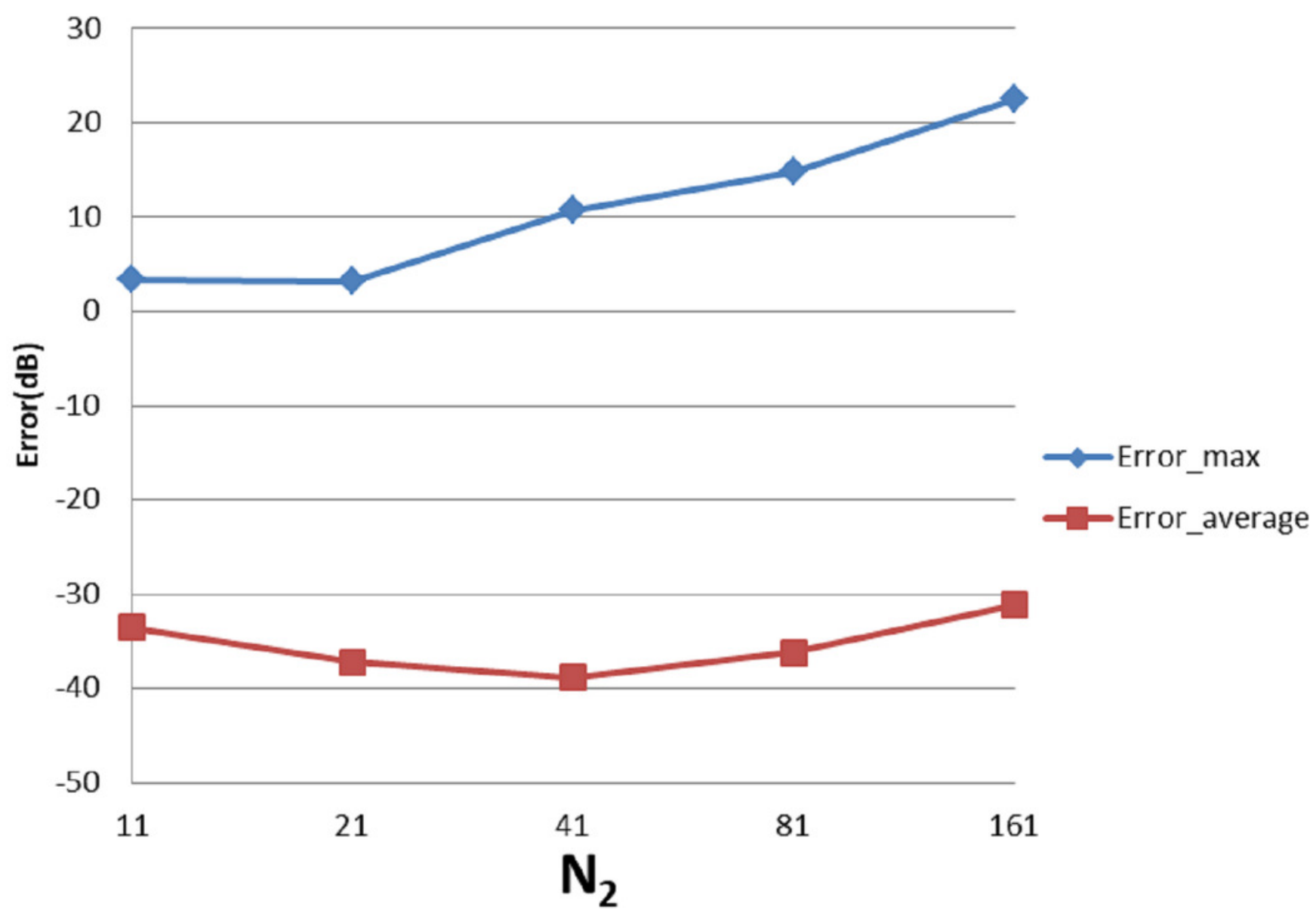




\section{Figure 20}

Error results for the $2 \mathrm{D}$ inverse discrete Fourier transform of the Four-term sinusoid and sinc function for $\mathrm{W}_{\mathrm{p}}=90$ and $\mathrm{N}_{1}=430$

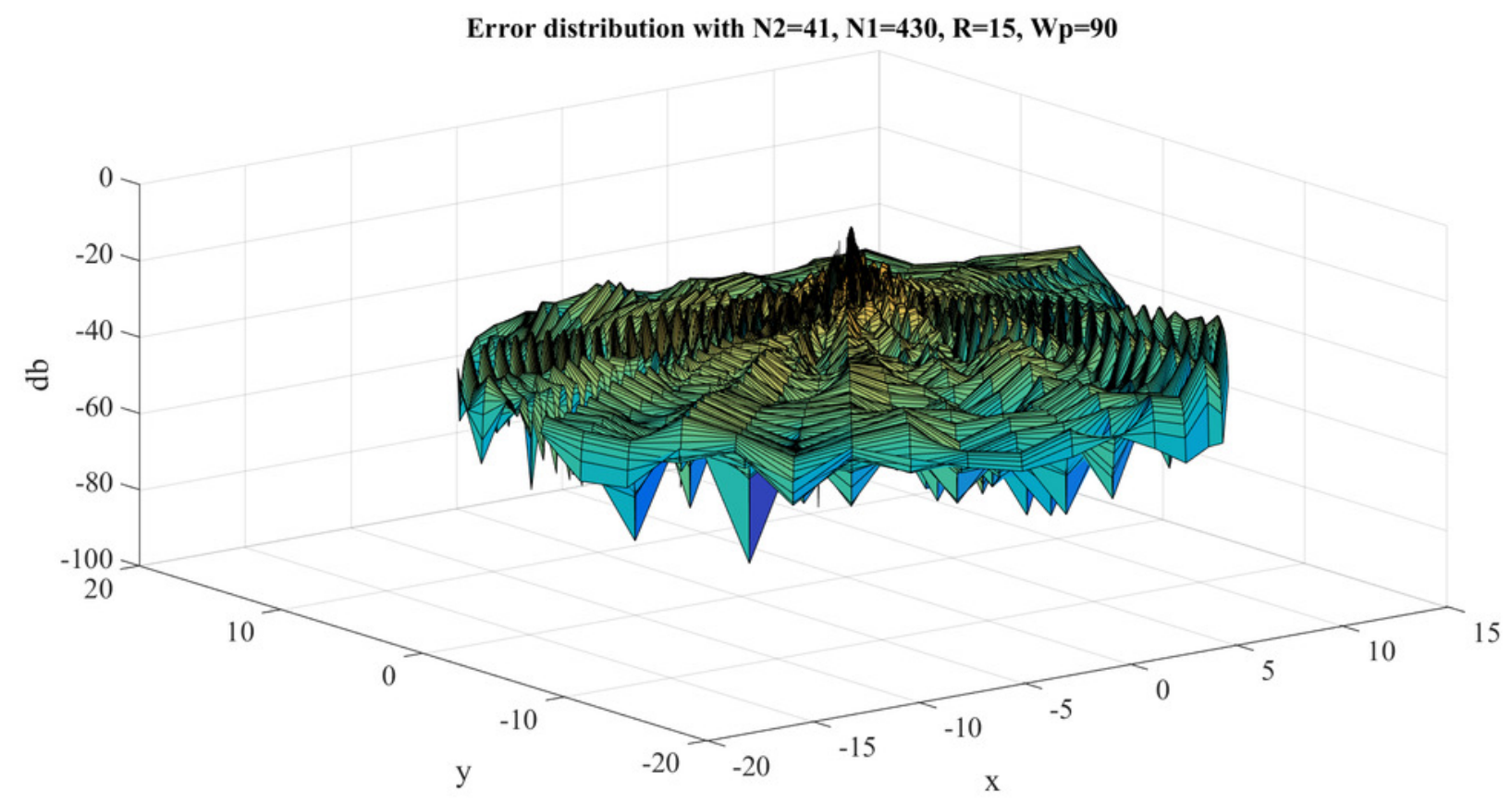


Figure 21

Error trend between the sampled values of the continuous inverse transform and the discretely calculated values for a four-term sinusoid and sinc function, as a function of $\mathrm{N}_{1}$

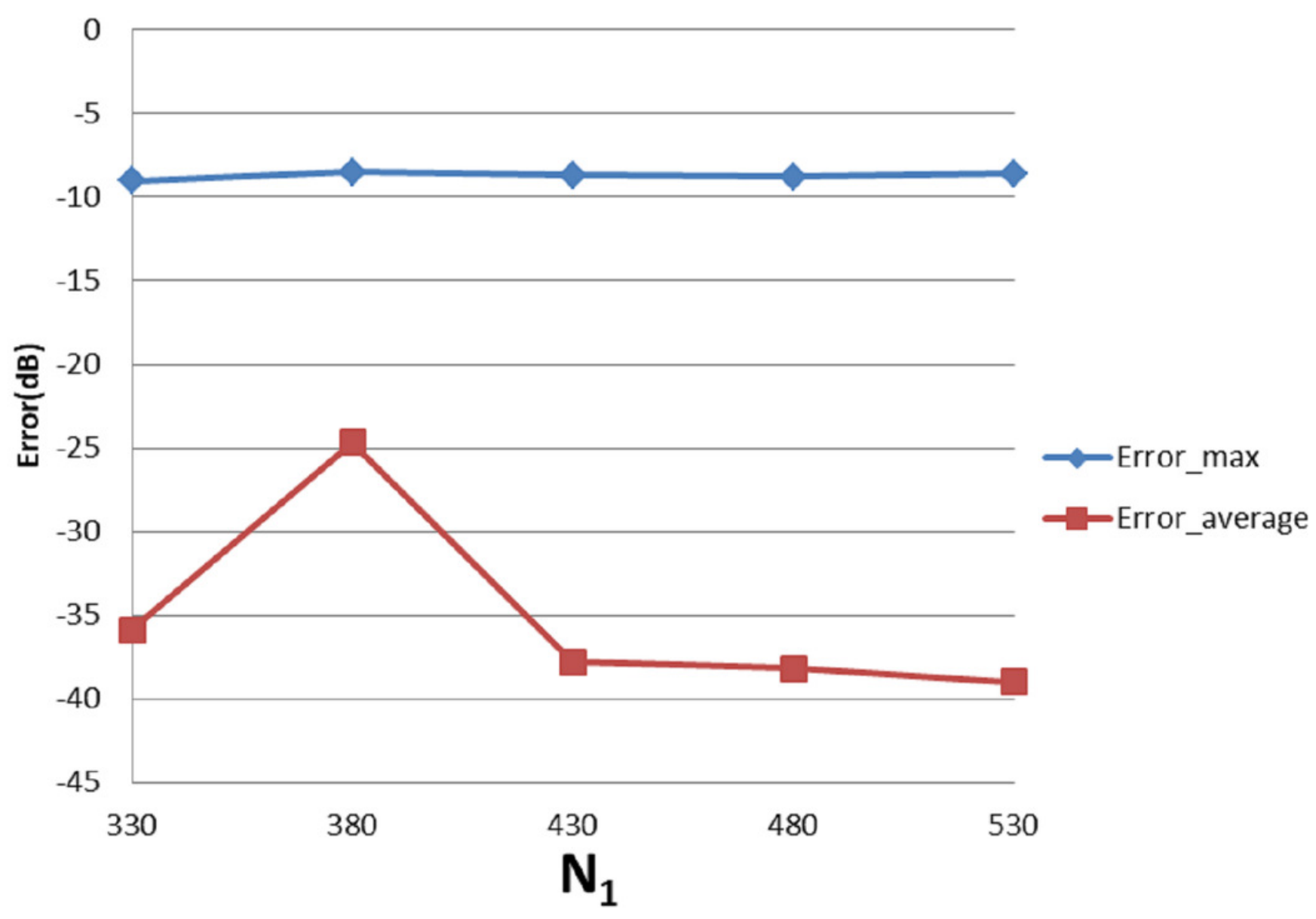


Figure 22

Error trend between the sampled values of the continuous inverse transform and the discretely calculated values for a four-term sinusoid and sinc function, as a function of $\mathrm{N}_{2}$

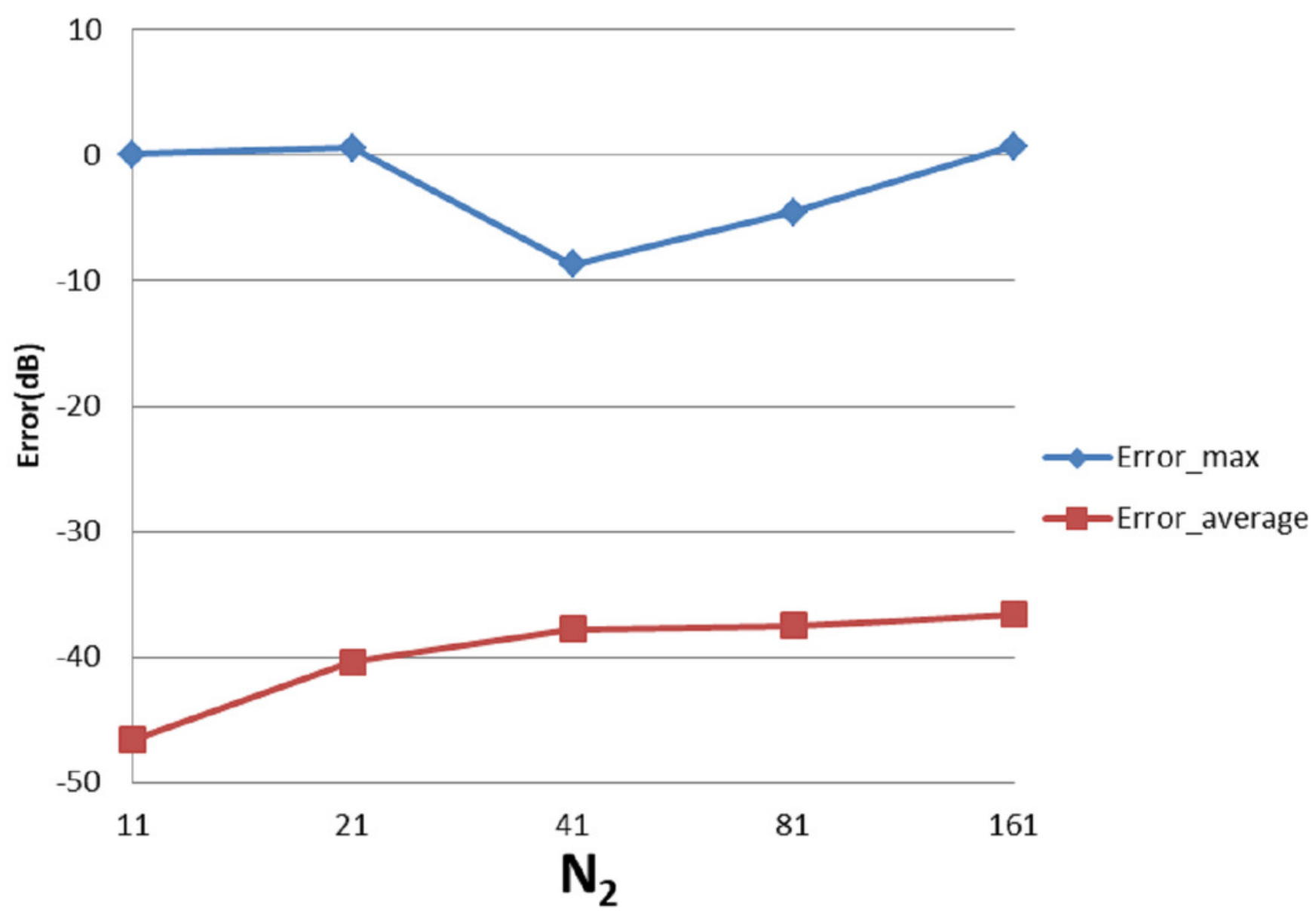




\section{Figure 23}

Plots for (a) the original function and (b) the magnitude of its continuous 2D discrete Fourier transform with $\mathrm{a}=0.1$ for a four-term sinusoid and modified exponential function

(a) Original Function

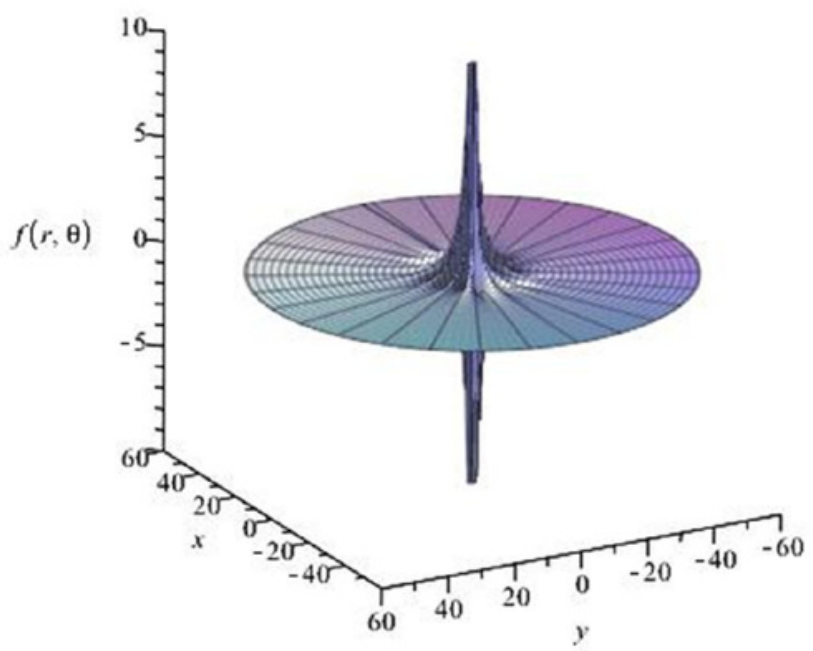

(b) 2D-Fourier Transform

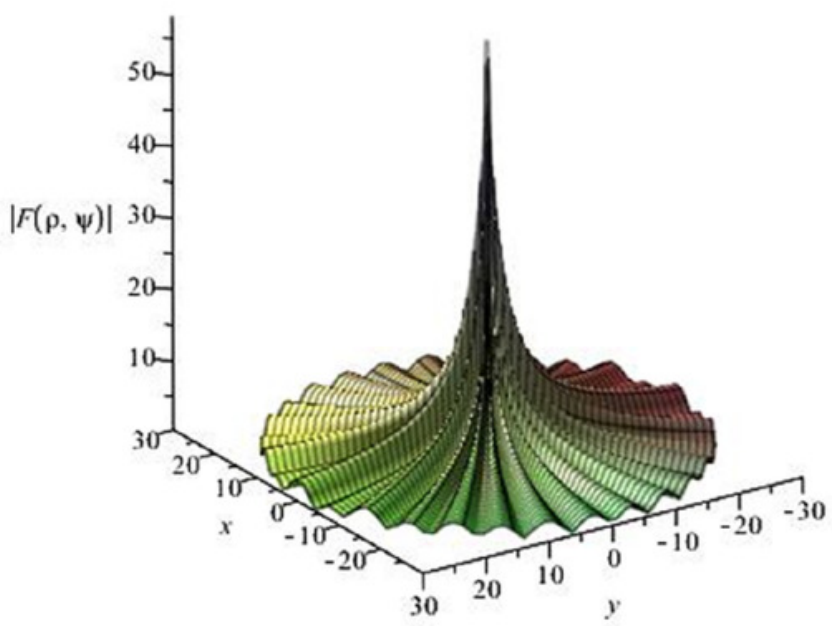




\section{Figure 24}

Error between the sampled values of the continuous forward transform and the discretely calculated values for the four-term sinusoid and modified exponential function with $\mathrm{R}=40, \mathrm{~W}_{\mathrm{p}}=30$ and $\mathrm{N}_{1}=383$

Error distribution with $\mathrm{N} 2=41, \mathrm{~N} 1=383, \mathrm{R}=40, \mathrm{Wp}=15$

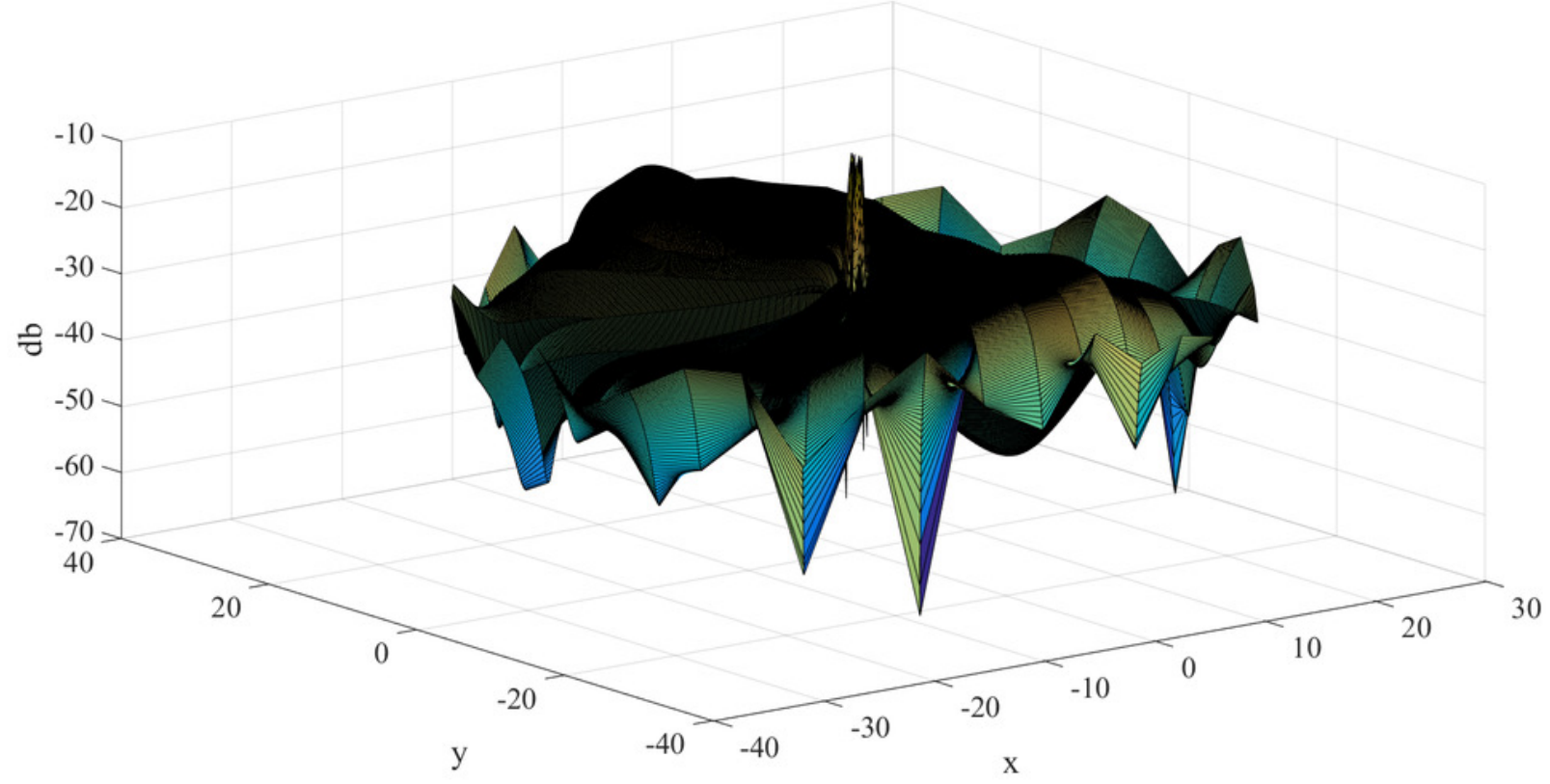




\section{Figure 25}

Error between the sampled values of the continuous inverse transform and the discretely calculated values for the four-term sinusoid and modified exponential function with $\mathrm{R}=40, \mathrm{~W}_{\mathrm{p}}=30$ and $\mathrm{N}_{1}=383$

Error distribution with $\mathrm{N2}=41, \mathrm{~N} 1=383, \mathrm{R}=40, \mathrm{Wp}=15$

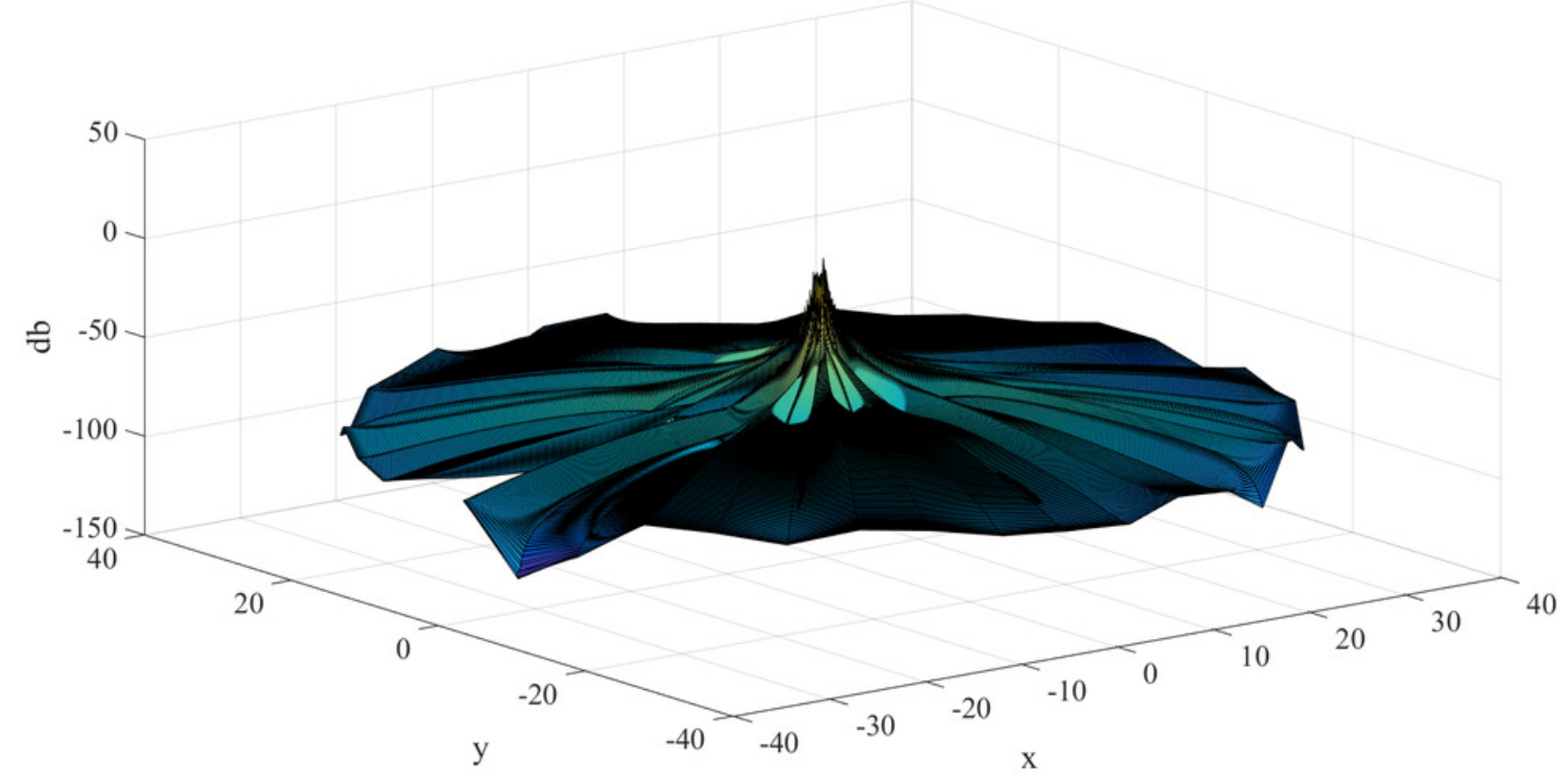




\section{Table $\mathbf{1}$ (on next page)}

Spatial grid coverage, $A_{r}$, with respect to different values of $N_{1}$ and $N_{2}(R$ is fixed) 
1

Table 1 Spatial grid coverage, $A_{r}$, with respect to different values of $N_{1}$ and $N_{2}$ ( $R$ is fixed)

\begin{tabular}{|c|c|c|c|c|}
\hline $\mathrm{N} 1$ & 15 & 75 & 150 & 300 \\
\hline 15 & $A_{r}=98.48 \%$ & $A_{r}=99.92 \%$ & $A_{r}=99.98 \%$ & $A_{r}=99.99 \%$ \\
\hline 75 & $A_{r}=93.78 \%$ & $A_{r}=99.36 \%$ & $A_{r}=99.81 \%$ & $A_{r}=99.95 \%$ \\
\hline 151 & $A_{r}=90.14 \%$ & $A_{r}=98.42 \%$ & $A_{r}=99.46 \%$ & $A_{r}=99.84 \%$ \\
\hline 301 & $A_{r}=86.17 \%$ & $A_{r}=96.58 \%$ & $A_{r}=98.59 \%$ & $A_{r}=99.51 \%$ \\
\hline
\end{tabular}

2

3 


\section{Table 2 (on next page)}

Frequency grid coverage, $A_{\rho}$, with respect to different values of $R$ and $N_{2}\left(W_{\rho}\right.$ is fixed) 
Table 1 Frequency grid coverage, $A_{\rho}$, with respect to different values of $R$ and $N_{2}$ ( $W_{\rho}$ is fixed)

\begin{tabular}{|c|c|c|c|c|}
\hline $\mathrm{R}$ & 15 & 75 & 150 & 300 \\
\hline 15 & $A_{\rho}=99.80 \%$ & $A_{\rho}=99.99 \%$ & $A_{\rho}=100.00 \%$ & $A_{\rho}=100.00 \%$ \\
\hline 75 & $A_{\rho}=97.66 \%$ & $A_{\rho}=99.91 \%$ & $A_{\rho}=99.98 \%$ & $A_{\rho}=99.99 \%$ \\
\hline 151 & $A_{\rho}=91.88 \%$ & $A_{\rho}=99.68 \%$ & $A_{\rho}=99.92 \%$ & $A_{\rho}=99.98 \%$ \\
\hline 301 & $A_{\rho}=70.67 \%$ & $A_{\rho}=98.83 \%$ & $A_{\rho}=99.71 \%$ & $A_{\rho}=99.93 \%$ \\
\hline
\end{tabular}

2 


\section{Table 3 (on next page)}

Error ( $d B$ ) of forward transform of Gaussian Function with $R=40$, di $\square$ erent value of $\mathrm{N}_{1}$ and $\mathrm{N}_{2}$ 
Table 1 Error (dB) of forward transform of Gaussian Function with $R=40$, different value of $N_{1}$ and $N_{2}$

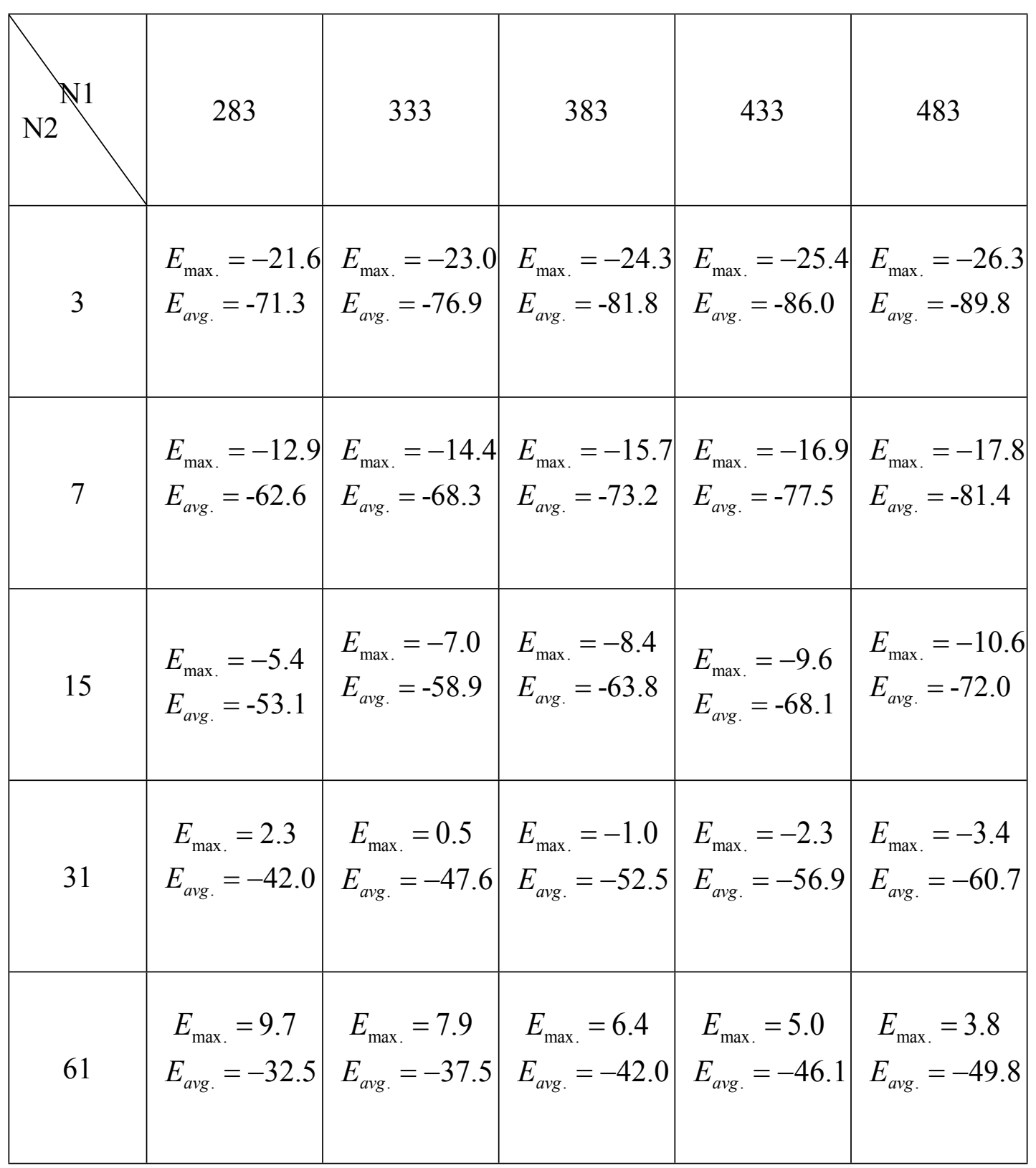




\section{Table 4 (on next page)}

Error ( $\mathrm{dB}$ ) of inverse transform of Gaussian Function with $\mathrm{R}=40$, di $\square$ erent value of $\mathrm{N}_{1}$ and $\mathrm{N}_{2}$ 
Table 1 Error (dB) of inverse transform of Gaussian Function with $R=40$, different value of $N_{1}$ and $N_{2}$

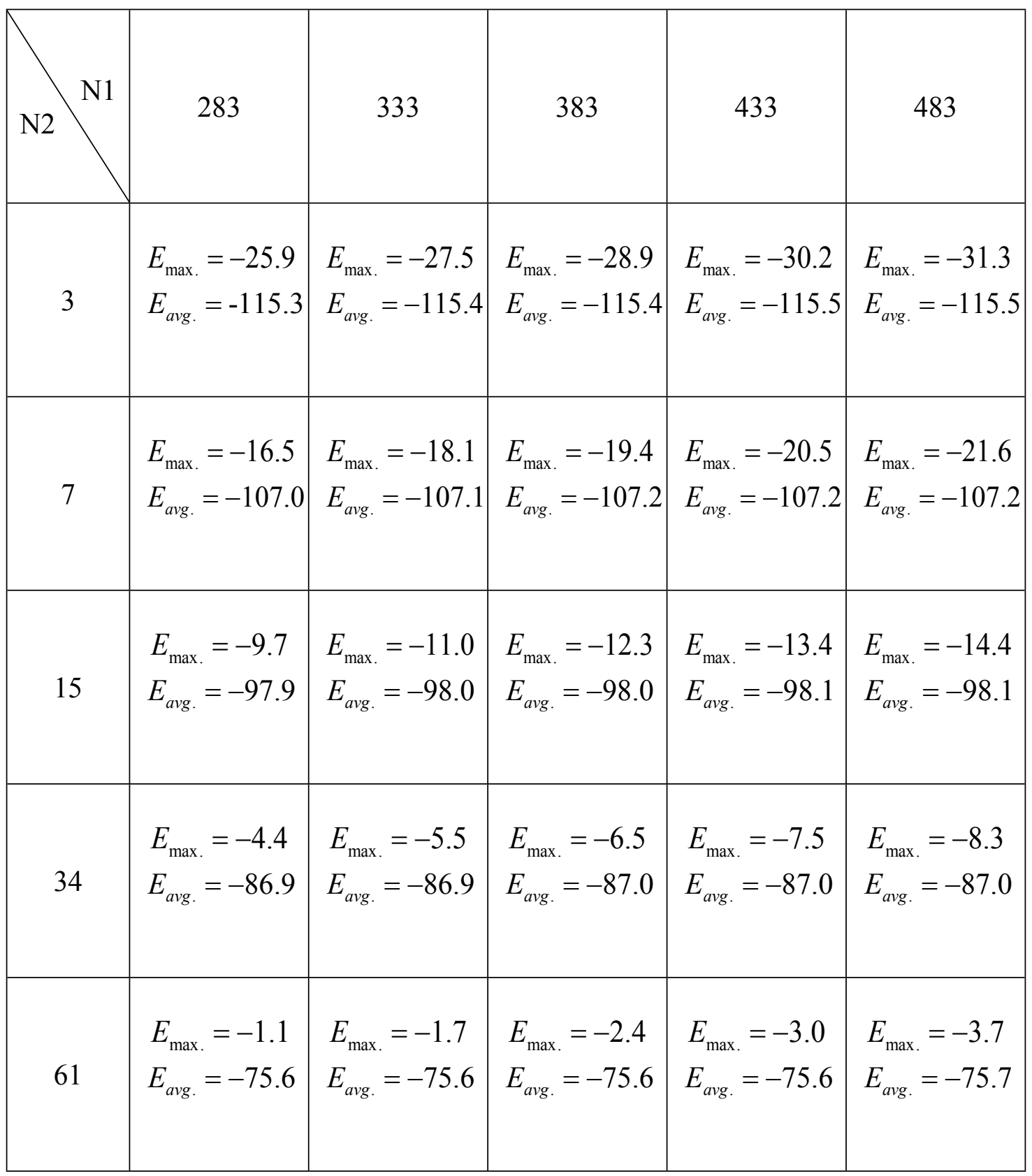




\section{Table 5 (on next page)}

Error $(\mathrm{dB})$ of the forward transform of $\square$ Four-term sinusoid \& Sinc $\square$ Function with di $\square$ erent value of $\mathrm{N}_{1}$ and $\mathrm{N}_{2}$ of forward transform 
Table 1 Error $(d B)$ of the forward transform of 'Four-term sinusoid \& Sinc' Function with different value of $N_{1}$ and $N_{2}$ of forward transform

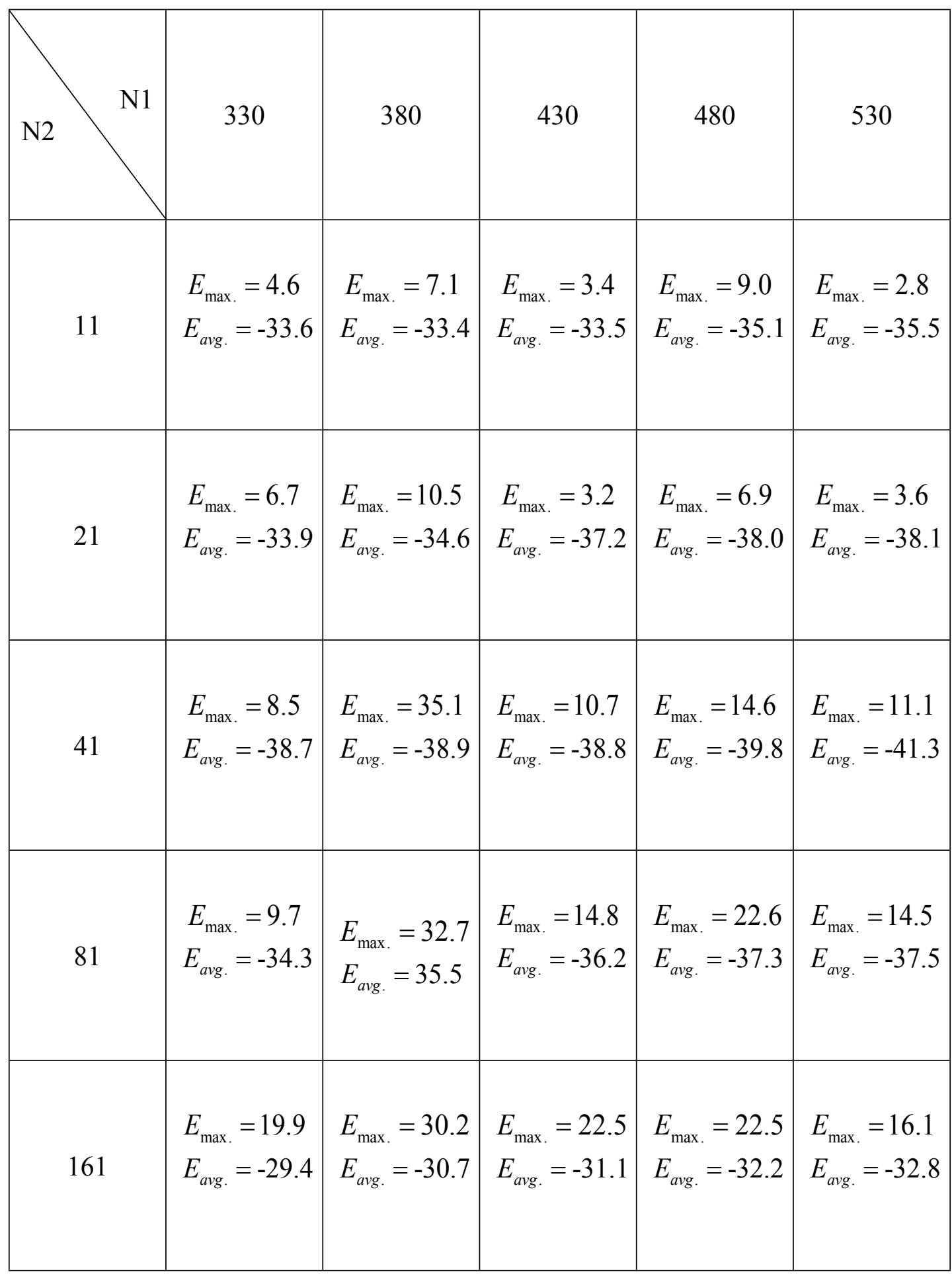




\section{Table 6(on next page)}

Error $(\mathrm{dB})$ of inverse transform of $\square$ Four-term sinusoid $\&$ Sinc $\square$ Function with di $\square$ erent value of $\mathrm{N}_{1}$ and $\mathrm{N}_{2}$ 
Table 1 Error (dB) of inverse transform of 'Four-term sinusoid \& Sinc' Function with different value of $N_{1}$ and $N_{2}$

\begin{tabular}{|c|c|c|c|c|c|}
\hline N2 & 330 & 380 & 430 & 480 & 530 \\
\hline 11 & $\begin{array}{c}E_{\text {max. }}=0.1 \\
E_{\text {avg. }}=-43.7\end{array}$ & $\begin{array}{c}E_{\text {max. }}=0.1 \\
E_{\text {avg. }}=-43.7\end{array}$ & $\begin{array}{c}E_{\text {max. }}=0.1 \\
E_{\text {avg. }}=-46.6\end{array}$ & $\begin{array}{c}E_{\text {max. }}=0.1 \\
E_{\text {avg. }}=-45.6\end{array}$ & $\begin{array}{c}E_{\text {max. }}=0.1 \\
E_{\text {avg. }}=-48.1\end{array}$ \\
\hline 21 & $\begin{array}{c}E_{\text {max. }}=0.7 \\
E_{\text {avg. }}=-38.3\end{array}$ & $\begin{array}{c}E_{\text {max. }}=0.7 \\
E_{\text {avg. }}=-38.0\end{array}$ & $\begin{array}{c}E_{\text {max. }}=0.6 \\
E_{\text {avg. }}=-40.4\end{array}$ & $\begin{array}{c}E_{\text {max. }}=0.6 \\
E_{\text {avg. }}=-40.6\end{array}$ & $\begin{array}{c}E_{\text {max. }}=0.7 \\
E_{\text {avg. }}=-42.2\end{array}$ \\
\hline 41 & $\begin{array}{l}E_{\text {max. }}=-9.0 \\
E_{\text {avg. }}=-35.9\end{array}$ & $\begin{array}{l}E_{\text {max. }}=-8.5 \\
E_{\text {avg. }}=-24.7\end{array}$ & $\begin{array}{l}E_{\text {max. }}=-8.7 \\
E_{\text {avg. }}=-37.8\end{array}$ & $\begin{array}{l}E_{\text {max. }}=-8.8 \\
E_{\text {avg. }}=-38.2\end{array}$ & $\begin{array}{l}E_{\text {max. }}=-8.6 \\
E_{\text {avg. }}=-39.0\end{array}$ \\
\hline 81 & $\begin{array}{l}E_{\text {max. }}=-4.5 \\
E_{\text {avg. }}=-35.7\end{array}$ & $\begin{array}{l}E_{\text {max. }}=-4.7 \\
E_{\text {avg. }}=-26.5\end{array}$ & $\begin{array}{l}E_{\text {max. }}=-4.5 \\
E_{\text {avg. }}=-37.5\end{array}$ & $\begin{array}{l}E_{\text {max. }}=-4.6 \\
E_{\text {avg. }}=-36.2\end{array}$ & $\begin{array}{l}E_{\text {max. }}=-4.5 \\
E_{\text {avg. }}=-39.0\end{array}$ \\
\hline 161 & $\begin{array}{c}E_{\text {max. }}=0.8 \\
E_{\text {avg. }}=-35.6\end{array}$ & $\begin{array}{c}E_{\text {max. }}=0.7 \\
E_{\text {avg. }}=-32.5\end{array}$ & $\begin{array}{c}E_{\text {max. }}=0.7 \\
E_{\text {avg. }}=-36.6\end{array}$ & $\begin{array}{c}E_{\text {max. }}=0.7 \\
E_{\text {avg. }}=-37.2\end{array}$ & $\begin{array}{c}E_{\text {max. }}=0.7 \\
E_{\text {avg. }}=-39.2\end{array}$ \\
\hline
\end{tabular}




\section{Table 7 (on next page)}

Computing time of three cases: Case1: Run the transform as matrixes in matrix without pre-calculating the Bessel zeros; Case2: Run the transform as DFT,DHT and IDFT in sequence without pre-calculating the Bessel zeros; Case3: Run the transform as DFT,DHT 
1 Table 1 Computing time of three cases: Case1: Run the transform as matrixes in matrix without pre-calculating the Bessel zeros;

2 Case2: Run the transform as DFT,DHT and IDFT in sequence without pre-calculating the Bessel zeros; Case3: Run the

3 transform as DFT,DHT and IDFT in sequence with pre-calculating the Bessel zeros

\begin{tabular}{|c|c|}
\hline Test cases & Total computing time(seconds) \\
\hline Case 1 & 3346.5 \\
\hline Case 2 & 321.1 \\
\hline Case 3 & 14.3 \\
\hline
\end{tabular}

4 